\title{
1 RBPSpot: Learning on Appropriate Contextual Information for RBP Binding
}

\section{Sites Discovery}

3 Nitesh Kumar Sharma ${ }^{1,2}$, Sagar Gupta ${ }^{1}$, Prakash Kumar ${ }^{1,2,3}$, Ashwani Kumar ${ }^{1}$, Upendra

4 Kumar Pradhan ${ }^{1,2,3}$, Ravi Shankar*1,2

5

6

$8{ }^{1}$ Studio of Computational Biology \& Bioinformatics,

9 CSIR-Institute of Himalayan Bioresource Technology (CSIR-IHBT),

10 Palampur (HP), 176061, India.

11

$12{ }^{2}$ Academy of Scientific and Innovative Research (AcSIR),

13 Ghaziabad, Uttar Pradesh- 201002

15 3 ICAR-Indian Agricultural Statistics Research Institute

16 Library Avenue, Pusa, New Delhi, Delhi 110012

20 *Corresponding Author: ravish@ihbt.res.in 


\section{Abstract}

28 Identifying RBP binding sites and mechanistic factors determining the interactions remain a big

29 challenge. Besides the sparse binding motifs across the RNAs, it also requires a suitable sequence

30 context for binding. The present work describes an approach to detect RBP binding sites while

31 using an ultra-fast BWT/FM-indexing coupled inexact k-mer spectrum search for statistically

32 significant seeds. The seed works as an anchor to evaluate the context and binding potential using

33 flanking region information while leveraging from Deep Feed-forward Neural Network (DNN).

34 Contextual features based on pentamers/dinucloetides which also capture shape and structure

35 properties appeared critical. Contextual CG distribution pattern appeared important. The developed

36 models also got support from MD-simulation studies and the implemented software, RBPSpot,

37 scored consistently high for the considered performance metrics including average accuracy of

$38 \sim 90 \%$ across a large number of validated datasets while maintaining consistency. It clearly

39 outperformed some recently developed tools, including some with much complex deep-learning

40 models, during a highly comprehensive bench-marking process involving three different data-sets

41 and more than 50 RBPs. RBPSpot, has been made freely available, covering most of the human

42 RBPs for which sufficient CLIP-seq data is available (131 RBPs). Besides identifying RBP binding

43 spots across RNAs in human system, it can also be used to build new models by user provided data

44 for any species and any RBP, making it a valuable resource in the area of regulatory system studies. 


\section{Introduction}

54 It has been reported that at any given time, compared to just 2-3\% transcription factors expression

55 share, 10 times higher volume of RNA binding proteins are expressed (1). Advances with high-

56 throughput techniques like CLIP-seq and Interactome Capture have drastically revised our

57 understanding about RBPs which suggest that human systems are expected to have at least 1,500 -

582,000 genes coding for RBPs $(1,2)$. Unfortunately, we are still far behind in terms of information for

59 these regulators where hardly 150 RBPs have been studied so far for their interactions with RNAs.

60 Despite of their critical functional roles in cell systems, very few RBPs have been explored with

61 precise identification of their mechanism of action (1).

63 There are certain limitations with these high-throughput experiments. These experiments are costly.

64 They too don't give the entire RBP-RNA interactome spectrum and at a time work for one RBP only

65 in condition specific manner. The CLIP-seq reads provide narrowed down regions to look for

66 interactions but don't provide the mechanistic details and explanations for the interactions. Using

67 general motif discovery tools to identify the interaction spots have got limited success in case of

68 RBPs as they either report too short motifs which have high chances of occurrences across the

69 random data or they don’t cover large spectrum of instances. Unlike transcription factors, RBPs

70 binding sites display sparse motif positional conservation. They are usually difficult to detect

71 through such routine motif finding approaches. Besides the binding motifs, contenxtual sequence

72 environment also guide the RBP-RNA interactions, adding further complexity to the process of

73 discovery of the actual interaction spots. Therefore, this is an area which needs prime focus on

74 deriving the principles of RBP-RNA interactions and their impact of regulation once we have 
enough CLIP-seq data. One of the most remarkable work, RNAcompete, was done where the authors identified in-vivo motifs for 207 different RBPs using pools of 30-41 bases long RNA oligos to which affinity of various RBPs was assessed for binding (3). RNAcompete also

81 highlighted how conventional motif finding tools fail to discover the binding sites motif for RBPs.

82 At computational front some decent progress has been made in dealing with these CLIP-seq data to

83 derive the models for interactions. Initially, to explore the RBPs and their RNA binding sites, 84 databases like RBPDB, CLIPZ, CLIPdb/POSTAR came up (4-7). These databases provided first 85 structured information on RBP-RNA interactions as well as proposed their interaction motifs using traditional motif finding tools while building on publicly available experimental data. As already mentioned above, the motifs being used here are short and occur in abundance even in random data. Also, they don't consider contextual information. Identification of correct RBP:RNA interaction motifs is a critical step which helps in locating the appropriate contextual information to build an accurate model of RBP:RNA interactions.

92 RNAcontext is among those first such tools which considered contextual information for RBP-RNA

93 interaction discovery. It applied the structural preferences information for these RNAcompete

94 motifs using ab-initio RNA structure prediction tool, sfold (8). However, these $a b$-initio structural 95 prediction methods reliability falls down with the length, making the structural information derived 96 through them not reliable enough (9). The next important stride came with probabilistic tools like RBPmap which extended their previous approach to identify splice sites while applying user provided position specific scoring matrices, supported motif clusters, and phylogenetic conservation to identify RBP RNA interaction spots (10). In the same probabilistic tools category, mCarts was

100 another important addition (11). It works on the similar lines to RBPmap but also applies 6-states 101 Hidden Markov Model (HMM) along with structural information from ab-initio secondary structure 102 prediction methods to predicted functional RBP binding sites. 
104 With Graphprot a new generation of such tools started which applied machine learning as well as

105 leveraged from new data-sets developed from CLIP-seq experiments (12). It also applied the concept of differential RNA secondary structure information in contextual manner to build the interaction models. A recently develop tool, beRBP, carries forward the approach similar to RBPmap while implementing a machine-learning method of Random-Forest (13). It clusters the potential motif sites where it ranks them and uses the highest scoring regions for the matches in the given region while scanning for the user provided motif/PWM. In the followup, they have also applied an approach similar to RNAcontext where RNA structural information is provided for the motif region using ab-initio structure prediction tool, RNAfold. Further to this, it added the 113 phylogenetic conservation information similar to RBPmap and mCarts.

115 With recent developments in the area of deep learning, many deep-learning based RBP-RNA interaction detection approaches have been implemented recently. DeepBind deserves special mention among them as it pioneered this category where a robust general system was created to model nucleic acids and protein interactions using convolution neural network (CNN) (14).

119 DeepBind has become a sort of prototype for almost all of the recent Deep-learning based tools to 120 identify the RBP-RNA interactions. DeepBind applies 7-mer motif weight matrices are 121 transformation into an image pixel matrix and is scanned for entire sequence while evaluating for 4122 stages to derive the binding score: convolution stage, rectification stage which zooms the scanner to 123 most promising regions for the motif, followed by pooling of all such regions and expansion and 124 clustering of motifs, which is finally subjected to a non-linear classifier. However, the authors 125 accepted that compared to transcription factors and their data, running DeepBind with RNAcompete 126 data did not achieve that level of accuracy. They pointed out the importance of accurate RNA 127 secondary structure information and RNA shape readouts in RNA-RBP interactions which most of 
128 the approaches have missed so far. Taking the work further on Deep-learning based RBP-RNA

129 interaction detection, another prominent tool system is iDEEP which has come like a series of 130 softwares like iDeep, iDeepS, and iDeepE (15-17). These tools differ from each other for the way

131 they applied various combinations of CNN and RNN layers. iDeepS applied CNN with Long-Short

132 term memory (LSTM) while taking input from sequence and RNAshape data. iDeepE applies

133 combinations of CNNs which capture local and global sequence properties. A recently developed

134 tool, DeepRiPe, has evolved a CNN and GRU based deep-learning approach while also introducing

135 transcript's regions specific information like splice junctions etc (18). DeepCLIP is another recently

136 developed tool which detects RBP-RNA interaction spots while applying CNN in combination with

137 bidirectional-LSTM and claims to detect sequence position specific importance which could

138 determine the contribution of various nucleotides in RBP binding (19). These very recently

139 developed deep-learning approaches have become much more complex than DeepBind and claim to

140 achieve much higher accuracy. Their complexity comes from adding complex layers above the

141 regular dense hidden layer. These complex layers actually do the job of automatic feature extraction

142 unlike the other machine-learning approaches where expert knowledge is applied to identify the

143 important properties to look into for feature extraction.

145 While reviewing these developments and tools, it looked imminent that there is an enormous scope 146 of improvement in the approaches to find and locate RBP-RNA interaction spots. Some of the major 147 points to consider would be: 1) Choice of datasets: A notable issue with all these algorithms is the 148 choice of data-sets, especially the negative data-sets, which have mostly been too relaxed and 149 unrealistic, due to which these tools are prone to over-fitting and imbalance. They are either 150 randomly shuffled sequences or regions randomly selected from those RNAs which did not bind the 151 given RBP. 2) Motif searching approach: most of existing tools, with exception of recent deep152 learning based approaches, begin with predefined/user defined motif or PWM derived from 
153 traditional motif finding tools with user defined length, which is not a natural approach and one of

154 the prime mistakes. RBP binding sites display sparse conservation which regular motif discovery

155 tools may fail to capture sufficiently. Third, high dependence on $a b$-initio RNA structure prediction

156 tools to derive the structural and accessibility information may be misleading, as already pointed

157 out above, such tools don't provide correct information on actual complete RNA length. A better

158 approach has been consideration of dinucleotide densities for such purpose $(20,21)$. Consideration

159 of RNA-shape appears very much important as pointed out by DeepBind as well as some other

160 recent works $(14,22,23)$. It has been reported that pentamers capture the essence of nucleic acid's

161 shape accurately (24), making them a suitable candidate to be evaluated along with dinucleotide

162 densities to derive RNA structure and shape information. Fourth, though the recent deep-learning

163 approach claim good success through automation of the process of feature extraction at the cost of

164 added complexity, the effectiveness of such automated feature detection needs to be evaluated.

165 Simpler models, if trained with carefully selected properties, are capable to outperform complex

166 models. This is why some of the shallow learning methods have outperformed deep-learning

167 methods on structured data (25, https://towardsdatascience.com/the-unreasonable-ineffectiveness-

168 of-deep-learning-on-tabular-data-fd784ea29c33) .

170 Considering these all factors, here we present a reliable Deep Neural Net (DNN) based approach to

171 build the mechanistic models of RBP-RNA interactions using high-throughput cross-linking data

172 while considering data from 99 experiments and for 137 RBPs for human system. An ultrafast k-

173 mer spectrum search approach was used to identify the most important seed regions in the sequence

174 for contextual information derivation. Contextual information for 75 bases flanking regions around

175 the identified seed derived motif was extracted in the form of variable windowed position specific 176 dinucleotide, pentamers, and heptamers density based propensities. The combined contextual

177 information was provided to a two hidden layers based dense feed-forward networks to accurately 
178 identify the RBP binding spots in RNAs. The developed models were used to identify the

179 interaction spots and scored very high accuracy with remarkable balance between sensitivity and

180 specificity as well as performance consistency when tested across a large number and different

181 types of experimental datasets. Molecular dynamics studies also supported these models. The

182 developed approach has been implemented as a freely available webserver and standalone software,

183 RBPSpot. It was comprehensively bench-marked across three totally unbiased standardized data-

184 sets for performance along with five recently published tools, including more complex deep-

185 learning based tools, where it outperformed all of them consistently across all these datasets for

186 most of the studied RBPs. Unlike most of the existing software which don't provide the option to

187 build new models from data, RBPSpot approach can be applied to detect human system RBP-RNA

188 interactions with its inbuilt models as well as it can be used to develop new models for other species

189 and new RBP data also.

191 Materials \& Methods

192 Data retrieval and processing

193 The study has considered human RBP models while using high-throughput sequencing data from

194 cross-linking experiments using various CLIP-seq techniques like CLASH, dCLIP, eCLIP, FLASH-

195 CLIP-seq, HITS-CLIP, iCLIP, PAR-CLIP, sCLIP-seq, uvCLAP-CLIP-seq. This data also includes

196 the two cell lines eCLIP data from ENCODE. Most of them are processed peak data collected for

197137 RBPs with starBase 2.0 as their primary source (26). A total of $872 \mathrm{Mb}$ peak data from 99

198 experiments were covered in this study for RBP-RNA interaction information from CLIP-seq

199 experiments (Supplementary Data 1 Sheet 1). The peak data of RBPs were downloaded in the form

200 of co-ordinates along with their associated RNA information on which they were binding. Peak data

201 were converted into BED file format along with their strand specificity information. Genome

202 sequences of human hg19 builds were obtained from UCSC browser. Peak data were also refined 
203 based on the length distribution and peaks laying in extreme range (length $>300$ bases and $<5$ bases)

204 were omitted from the study (Supplementary Data 1 Sheet 2).

Identification of motif seed candidates: k-mer spectrum search using BWT/FM-Indexing

207 To search binding sites motifs/seeds for any particular RBP, all the peak regions were transformed

208 into overlapping lists of k-mers of size six to start with. Iteratively and in parallel these generated k-

209 mer spectrum for each such sequence was searched across all the reported cross-linked associated

210 regions in the targets to obtain the enrichment status of the k-mers (seeds) on which motif would be

211 built. These searches were allowed with maximum 30\% mismatches. Since normal search would be

212 heavily time consuming step, we implemented an enhanced Burrow-Wheeler transformation with

213 FM-Indexing to search with any number of mismatch which made the search ultra-fast for even in-

214 exact searches. The detailed algorithmic implementation pseudo-code of the implemented algorithm

215 is given in the supplementary methods.

217 Identification of motif seeds candidates: Anchoring with the significant seeds

218 All the k-mer seeds and their relatives displaying at least $70 \%$ similarity were evaluated for 219 existence across at least 70\% of peak data. Such motif seed candidates were further evaluated for 220 their statistical significance. Those RBPs where no k-mer and their relatives crossed $70 \%$ 221 representation were looked for the highest representation available. The remaining data which did 222 not show the representative k-mer were checked further and recursively with minimum cut-off of 223 20\% data representation. Motifs coming from such data were considered as mutually exclusive one.

224 Null model distribution probabilities of occurrence of each k-mer along with its relatives was 225 calculated from the random data set to find their random probabilities. Random data set was 226 generated from unassociated RNAs while randomly carving out the lengths similar to the peak data. 
227 Significantly over-represented k-mers were screened using binomial test with p-value cut-off of

228 0.01. These significantly enriched k-mers were used as initial seeds to develop the final motif.

229 These seeds of significantly enriched k-mers were expanded in both the directions by expanding by

230 one nucleotide both sides, followed by search across the peak data with at least $70 \%$ occurrence in

231 the peak data while repeating the above mentioned search operation recursively. Expansion of seed

232 region in both directions was allowed till at least 70\% match existed. Final motifs were selected on

233 the basis of satisfying both the criteria i.e. the motif displayed least $70 \%$ abundance across the

234 CLIP-seq instances at 1\% significance level and the maximum k-mer expansion maintained at least

$23570 \%$ identity with the associated sequences and relatives. Mutually exclusive motifs were other

236 predominant motifs which existed in the remaining data which were scanned in similar recursive

237 manner as described above. Figure 1. shows the part of the k-mer based motif seed discovery and

238 steps taken afterwards. (Supplementary Data 1 Sheet 3,4)

\section{Datasets creation}

241 Once we had prime motifs anchored for each RBP from the given data, their associated peak data

242 sequences were converted into positive datasets. To generate positive datasets for each RBP, start

243 and end co-ordinates from the main motif's both terminals were expanded by +75 and -75 bases

244 into both the directions. In case of multiple motif locations originating from a single peak for the

245 main motif, all the locations were expanded. Different length dataset sequences formed for different

246 RBPs which depended mainly upon the length of the core motif region. However, for any single

247 RBP all the sequences of the dataset were of same length.

249 To generate the negative datasets for each RBP, similar condition corresponding RNA-seq data were 250 downloaded from GEO. With minimum three replicates of RNA-seq data expression of each RNA 
251 was calculated. Only those transcript sequences were considered which had expression condition

252 available for the same condition but did not bind the RNA or which was not found present in the 253 corresponding condition's CLIP-seq binding data. Associated main motifs for the RBP were

254 searched across these RNA sets also just in the similar manner as was done to the positive dataset 255 instances. Locations of the main motif were reported in the form of start and end co-ordinates from 256 where further expansion of +75 and -75 bases was done on both the sides. This way very strong 257 negative data-sets were built which ensured that learning was in no way influenced by the motif 258 alone as the motif may also occur randomly to some extent and surrounding context is also 259 considered along in a right manner. This approach was carried out for 74 RBPs for which similar 260 condition RNA-seq data were available. Datasets derived this way were called Set A data-sets.

262 For 57 RBP similar RNA-seq data were not available for the corresponding conditions. In such 263 scenario the main motifs for negative datasets were searched in those regions which did not appear 264 in the CLIP-seq data but belonged to the same target RNA sequences whose some part appeared in 265 the CLIP-seq, suggesting that though the RNA expressed and even bound to the RBP, these regions 266 despite of having the motif for the RBP did not bind to the RBP and may work as a suitable 267 negative dataset. +75 and -75 flanking bases from both the terminals of the motifs were considered 268 along with the motif region to build the negative datasets. These data-sets were called Set B data269 sets.

\section{Feature generation for positive and negative datasets}

272 Five different types of properties were considered for input into machine learning: 1) The main 273 motif itself, 2) Di-nucleotide density in the associated region while considering 75 bases flanking 274 regions from both the sides of the motif, 3) Dot bracket representation of the RNA structural triplet 
275 for the data-set sequences, covering twenty seven combinations of structure triplets arising from the

276 dot-bracket structural representation from RNAfold predicted RNA structures [.((, .(), .(., .)

$(,).),.) ., . .(, .),. \ldots .,(((,((),((.)(),(,()),() .,(.(,(),.(. .),(())()),(., ~))()))$, ))., ).(, ).), ).., ] , 4) Pentamers

278 density profile for each position which captures the shape information, and, 5) Heptamers densities

279 for the complete region. Dinucleotide densities were evaluated for their discriminatory power for

280 multiple sliding windows starting from 17 to 131. Similarly, the dot brackets structural triplets

281 representation of the data-set sequences were generated using RNAfold (27). They too were

282 evaluated for optimum windows size while testing for window sizes ranging from 29 to full

283 sequence. 1,024 pentamers and 16,384 heptamers densities were evaluated in the similar manner

284 across the data-set sequences.

286 To calculate heptamers based feature, all positive datasets were split into k-mers of seven bases.

287 Probability of each k-mer were calculated with maximum of two mismatches for each position and

288 accordingly populated in the tensor. Thus, we had 16,384 X ((sequence length) - 7) tensor of 289 probabilities. 16,384 rows represent the heptamers and 150 columns represent individual positions.

290 In the similar manner pentamer features were calculated. For that we had $1024 \mathrm{X}$ ((sequence

291 length)-5) tensor of probabilities. These both tensors were used to convert the sequence data into

292 vectors of probabilities. All together, based on optimum windows, the combined features sets

293 representation of all the data-set sequences was done. The optimum windows and total features

294 varied for each RBP. Finally, each data-set was broken into training and testing data-sets ensuring

295 that no instances from training ever appeared in the testing data-sets. The breakup for each RBP for

296 their training and testing data-sets is given in Supplementary Data 1 Sheet 5 and 6. 
299 After generating all the features from positive and negative data-sets these features were

300 individually checked for their performance using tree based approaches which are expected to

301 perform better on high dimension instances. Random forest and XGBoost were applied. Each

302 property and their associated feature sets were evaluated for the varying window sizes for their

303 discrimination power between the positive and negative sets. Sliding windows of variable sizes

304 were used for dinculeotide and structure based features. These variable sizes windows were

305 evaluated for the performance. Out of these different sized windows the size producing the best

306 performance was kept for final model generation. It was found that the best performing window size

307 varied across the RBPs, resulting into different optimum windows for the RBPs.

309 Pentamers and heptamers appeared most informative on the full length window. Equal number of

310 positive and negative instances were chosen for all RBPs considered in the study. From the total

311 chosen instances, $60 \%$ were used to create the training set, while remaining $40 \%$ instances were

312 used to create the testing set. Python scikit-learn library was used for the same purpose. For feature

313 importance evaluation F-score was used for every considered feature. F-score locates the features

314 which display major difference between their values between negative and positive training sets

315 while comparing the averages for the feature values for positive, negative, and whole set of

316 instances (28). The F-score is represented by the following equation:

$$
F(i)=\frac{\left(\left(\bar{x}_{i}\right)^{+}-\overline{x_{i}}\right)^{2}+\left(\left(\overline{x_{i}}\right)^{-}-\bar{x}_{i}\right)^{2}}{1 /\left(n_{+}-1\right) \sum_{k=1}^{n^{+}}\left(x_{(k, i)}^{+}-\overline{\left(x_{i}\right)^{+}}\right)^{2}+1 /\left(n_{-}-1\right) \sum_{k=1}^{n^{-}}\left(x_{(k, i)}^{-}-\overline{\left(x_{i}\right)^{-}}\right)^{2}}
$$

319 Where:

$320 \mathrm{~F}(i)=$ Feature score for the ith feature, 
$321\left(\bar{x}_{i}\right)^{+}=$Avergae for i-th feature across the positive instances

$322 \overline{x_{i}}=$ Total average of the i-th feature across the complete data-set

$323\left(\overline{x_{i}}\right)^{-}=$Avergae for i-th feature across the negative instances

$324 x_{(k, i)}^{+}=$Feature value for $k$-th instance for $i$-th feature in positive data-set

$325 \overline{X_{(k, i)}}=$ Feature value for $k$-th instance for $i$-th feature in negative data-set

$326 n_{+}=$Total number of positive instances

$327 n-=$ Total number of negative instances

328 Also, for every $i$-th feature, t-test was conducted between $n_{+}$and $n_{-}$to evaluate the significance of $i$ -

329 th feature for its discrimination capability between positive and negative instances.

\section{Machine learning implementation}

332 With the optimized windows in the above mentioned section, feature vectors for all the RBPs were

333 used to build models to recognize RBP binding sites using two major machine-learning approaches:

334 XGBoost and Two Hidden Layers based Deep Feed Forward Neural Networks (DNNs). Both were

335 implemented using python scikit-learn, Keras, and Tensorflow libraries. In both the cases $70 \%$ and

$33630 \%$ of data were retained for train and test sets, respectively.

338 The DNNs were built where the input layers had number of nodes equal to the number of features

339 for the RBP considered. Thus, the size of input layer varied from 1,200 to 2,500. The performance

340 of DNN was also evaluated for various numbers of hidden layers where finally total two hidden

341 layers were found performing the best. The connections between the nodes were made dense. For 
342 every RBP model the number of nodes across the two hidden layers varied between 700 to 1,300.

343 Different types of activation functions combinations were applied for the layers from a pool of a

344 number of available activation functions. Activation functions define the layers and transform the

345 activation values obtained from previous layer to a non-linear form, creating several hyperplanes to

346 obtain best possible discrimination of instances. In most of the models here, the first hidden layer

347 had RELU and the second hidden layer had ELU (for some cases they interchanged also), while the

348 final output layer had sigmoid function.

350 Every learning step provides estimation of error made, measuring the error and accordingly 351 corrections in the learning rate and weights on connections are done. This error estimation is 352 achieved by loss/cost functions. Multiple types of loss functions were tried to optimize the accuracy.

353 The best performance was obtained for Binary Cross Entropy. Since its a feed forward network 354 where the cost function assess the missed targets and accordingly network connection weights are updated though some optimizer. The optimizer parameter which worked the best was 'Adam' optimizer, otherwise SGD with momentum. Usually Adam optimizer works better because of its capability to provide different learning rates per parameter, deals better with sparse gradients, and adapts based on recent learning rates while keeping them in memory. Momentum was applied in the learning which helps to ward-off entrapment under local minima during the minimization steps. The learning rate varied from 0.001 to 0.01 and momentum varied from 0.05 to 0.9 . L1 and L2 weight decay regularizors were applied to avoid over-fitting. DNN models were trained using 1000 epochs and batch sizes varying from 50 to 200 instances. All the model from DNN and XGBoost were saved in protobuf format. Since the entire system is implemented here using TensorFlow, the protbuf file provides the graph definition and weights of the model to the TensorFlow structure. The optimum parameter values were fixed using an in-house developed script which tested various combinations of values of the paramters to pick the best ones. 
368 In XGBoost, grid search was applied for parameter optimization. Following parameters were

369 finalized after the grid search: params = \{"eta/learning rate": 0.2, "max_depth": 4, "objective":

370 "binary:logistic", "silent": 1, "base_score": np.mean(yt), 'n_estimators': 1000, "eval_metric":

371 "logloss"\}. Gradient boosted decision trees learn very quickly and may overfit. To overcome this

372 shrinkage was used which slows down the the learning rate of gradient boosting models. Size of the

373 decision tree were run on max-depth=9. At the value of 4 stability was gained as the logloss got

374 stabilized and did not change thereafter.

376 To evaluate the consistency of performance models developed with the given features, 10 -fold cross

377 validation was also performed for each RBP. Everytime, the training dataset was split into 70:30

378 ratio with first used to train and second part used to test, respectively. Each time data was shuffled

379 and random data was selected for building new model from scratch. This process was repeated 10

380 times for each RBP. Accuracy and other perfomance measure were calculated for each model.

381 (Supplementary data 1 sheet 7)

383 The performance on test sets was also evaluated. Confusion matrices containing correctly and

384 incorrectly identified test set instances were built for each RBPs. Frequently used measures for

385 classifier performance evaluation and accuracy of RBPs models were evaluated. Sensitivity

386 (Sn)/Recall/True Positive Rate (TPR) defines the portion of positives which were correctly 387 identified as positives whereas specificity describes the portion of negative instances correctly 388 identified. Precision estimates the proportion of positives with respect to total true and false 389 positives. F1-score was also evaluated which measures the balance between precision and recall. 390 AUC/ROC were also measured for each model. Besides these metrics, Mathew's Correlation 
391 Coefficient (MCC) was also considered. MCC is considered among the best metrics to fathom the

392 performance where score equally influenced by all the four confusion matrix classes (true positives,

393 false negatives, true negatives, and false positives) (29). A good MCC score is an indicator of robust

394 and balanced model with high degree of performance consistency.

395 Performance measures were done using the following equations:

$$
A c c=\frac{T N+T P}{(T N+T P+F N+F P)}
$$

Specificity $(\mathrm{Sp})=\frac{\mathrm{TN}}{(\mathrm{TN}+\mathrm{FP})}$

Precision $=\frac{\mathrm{TP}}{(\mathrm{TP}+\mathrm{FP})}$

Recall/Sensitivity $(\mathrm{Sn})=\frac{\mathrm{TP}}{(\mathrm{TP}+\mathrm{FN})}$

$F 1-$ Score $=2 \times\left(\frac{\text { Precision } \times \text { Recall }}{\text { Precision }+ \text { Recall }}\right)$

$\mathrm{AUC}=\int_{0}^{1} \operatorname{Pr}[\mathrm{TP}](v) \mathrm{dv}$

$\mathrm{MCC}=\frac{\mathrm{TP} \times \mathrm{TN}-\mathrm{FP} \times \mathrm{FN}}{\sqrt{(\mathrm{TP}+\mathrm{FP})(\mathrm{TP}+\mathrm{FN})(\mathrm{TN}+\mathrm{FP})(\mathrm{TN}+\mathrm{FN})}}$

403 Where:

$404 \mathrm{TP}=$ True Positives, $\mathrm{TN}=$ True Negatives, $\mathrm{FP}=$ False Positives, $\mathrm{FN}=$ False Negatives, Acc $=$

405 Accuracy, AUC = Area Under Curve

\section{Structural analysis of identified binding spots}

408 To assess the stability and dynamics of the RBP-RNA complexes for the identified binding spots, 409 structural analysis was done. The 3D coordinates of RBPs were retrieved from the Protein Data 
410 Bank (PDB). X-Ray crystallographic structure for 13 different RBPs were downloaded. Prior to

411 docking, protein structures were prepared by removing water molecules and other hetero-atoms,

412 while adding polar hydrogen atoms. RNA motifs identified through RBPSpot algorithm for above

413 mentioned five RBPs were taken as flexible molecules. All docking studies were performed through

414 NPDock (Nucleic Acid-Protein Docking) and PATCHDOCK incorporating more realistic DARS-

415 RNP statistical potential based on reverse Boltzmann statistics to score protein-RNA complexes

416 (30). RNA motifs three dimensional structures were built using RNACOMPOSER web server based

417 on RNA FRABASE database relating the RNA secondary and tertiary structure elements. In order

418 to search for all possible RNA-binding sites and optimize the structural effects of RNA on the

419 construction of complex, short RNA motifs were taken into account. Protein-RNA interface residues

420 were predicted using DR_Bind1 (31) based on evolutionary conservation. Top three representative

421 docking potential-ranked protein-RNA complexes were built for each of the above mentioned RBPs

422 and the best one was considered for further analysis.

\section{MD simulations}

425 All molecular dynamics simulations of the RBP alone and the RBP-RNA complex were conducted

426 using GROMACS 5.1 package (32), modeling each system with the AMBER03 force-field of 427 protein and nucleic acids (33) with periodic boundary conditions. The topology files for the selected 428 target RNA motifs were built using pdb2gmx in the framework of AMBER03 force-field. Models 429 were solvated with the TIP3P water model (34). The distance between the biomolecule and the edge 430 of the simulation box was set as minimum $1.0 \AA$ so that they could not directly interact with their 431 own periodic boundary condition and fully immerse with water while rotating freely. Boxes were 432 solvated with TIP3P water. The number of solvated molecules added to each system varied. After 433 the establishment of initial configuration, the systems were minimized. 50,000 steps ( steepest 434 descent approach) were used for each system until the maximum force of $<10.0 \mathrm{~kJ} / \mathrm{mol}$ for energy 
435 minimization. For calculation of long range electrostatic interactions, Particle Mesh Ewald (PME)

436 method was used. To establish the systems at constant temperature of 300K, V-rescale thermostat

437 (modified Berendsen thermostat), at a constant pressure of 1 bar, and Parrinello-Rahman berostat

438 were applied with a 2 ps coupling constant for both parameters. The LINCS algorithm (35) was

439 used to constrain all bond lengths involving hydrogens. During the production run, a time step of 2

440 fs was used and conformations were saved every 10 ps for the analysis of molecular dynamics

441 trajectory of total 20 ns for each RBP and their complexes using leap-frog algorithm (36) to

442 integrate the equation of motion. MD trajectories were further evaluated for considering Root Mean

443 Square Deviation (RMSD). RMSD is suitable to decipher the structural changes in proteins and

444 their complex structures corresponding to initial structure during the course of different time

445 periods of dynamics simulation. RMSD was calculated using the following equation:

$\mathrm{RMSD}=\overline{\frac{1}{N} \times \sum_{\mathrm{i}=1}^{N}\left|u_{i}-v_{i}\right|^{2}}$

447 where,

$448 \mathrm{u}_{\mathrm{i}}=$ Cartesian coordinates of atom $i$ in the initial structure;

$449 \quad \mathrm{v}_{\mathrm{i}}=$ Cartesian coordinates of atom $i$ in the structure during simulation;

$450 \quad \mathrm{~N}=$ number of atoms;

451 To analyze the structural properties of the individual RBPs and their complexes in the form of root 452 mean square deviation (RMSD), g_rms functions were utilized. Changes in trajectories of molecular 453 dynamics during course of simulation were plotted for evaluation using python plotting library. 
458 A two steps statistical approach was employed to identify the co-occurring motif pairs. In this

459 approach, the positive set of RBP was scanned for other most frequent occurring k-mers. Top co460 occurring motifs were checked for their statistical significance. KS-test was used to find the 461 significance of distance for two motifs. All the distance between two motifs were calculated from 462 positive and negative data-sets. Distribution plot of random data and positive data were further 463 checked using KS-test. Level of significance were considered $p<0.05$. They were further checked 464 for frequency ratio (FR). At 5\% level of significance, if the hypergeometric test $p$-value was less 465 than 0.05, motif pair of enriched and co-occurring motifs was considered significant. Additionally, 466 frequency ratio (FR) as a measure of co-occurrence of motif pairs was also computed to estimate 467 the tendency of motif pairs to co-occur with each other as proposed previously (37): $F R\left(\right.$ Motif $\left.{ }_{M 2 / M 1}\right)=\frac{X_{M 2 / M 1} / N_{M 1}}{Y_{M 2 / M 1} / M_{M 1}}$

$469 \mathrm{X}_{\mathrm{M} 2 / \mathrm{M} 1}=$ Number of sequences containing motif1

$470 \quad \mathrm{~N}_{\mathrm{M} 1}=$ Number of sequences containing motif2 co-occurring with motif1

$471 \quad \mathrm{Y}_{\mathrm{M} 2 / \mathrm{M} 1}=$ Number of sequences without motif1

$472 \mathrm{M}_{\mathrm{M} 1}=$ Number of sequences containing motif2 without motif

\section{Benchmarking and Performance Evaluation}

475 To evaluate the RBPSpot performance and the importance of dataset constructed in this study, we 476 compared RBPSpot with five different tool: RBPmap, DeepBind, iDeepE, DeepCLIP, and beRBP.

477 Three different datasets were considered separately for the benchmarking process: Datasets used for 478 RBPSpot, beRBP, and Graphprot. Datasets of beRBP and Graphprot are common data source for 479 most of the existing published software built to identify RBP-RNA interactions. As already 480 mentioned above, RBPSpot dataset is based on the positive datasets from ENCORI (the 481 encyclopedia of RNA Interactomes, previously known as StarBase) and the negative datasets based 
482 on the protocol mentioned above in the previous section. This dataset contained positive and

483 negative sequences for 131 RBPs in which length of sequence varied from minimum of 156 to

484 maximum of 160 bases. The variation in the length of the sequences for different RBPs was due to

485 the varying length of their major motifs. For benchmarking purpose those RBPs data were

486 considered from this dataset for which at least one tool had model ready for comparison. No such

487 RBP was considered from this dataset for benchmarking for which no other tool had model ready

488 for comparison. This way a total of 52 RBP data were used from RBPSpot dataset for the 489 comparison purpose.

491 The beRBP dataset is available for 29 RBPs. This dataset is based on the experimentally validated 492 target sequences (3'-UTRs) for human RBPs (positive datasets) from AURA (38) (v2, 493 8/5/2015;http://aura.science.unitn.it/), which is a manually curated and comprehensive catalog of 494 human UTRs bound by regulators including RBPs. Negative instances of this dataset has random 495 sequences chosen from the 3'-UTR pool. The beRBP dataset was obtained from the URL 496 http://bioinfo.vanderbilt.edu/beRBP/download/TabS1.7z.

498 The third dataset considered in this study was built during the work presenting Graphprot software.

499 Since then, this dataset has been used extensively by many published software to this date. This 500 dataset covers 24 RBPs coming from various CLIP-seq experiments. For each set of CLIP-seq data, 501 they created a set of unbound sites by shuffling the co-ordinates of bound sites within all genes 502 occupied by at least one binding site which worked as the negative dataset. making the 503 corresponding negative dataset instances. This dataset was retrieved from URL 504 http://www.bioinf.uni-freiburg.de/Software/GraphProt/GraphProt CLIP sequences.tar.bz2. 
506 The four out of the compared five tools viz. beRBP, RBPmap, DeepCLIP, and DeepBind provide

507 pre-built models. Only iDeepE does not provide any pre-built model. To overcome this, models

508 were generated using iDeepE methodology for the datasets. To make binary decisions with

509 DeepBind, threshold of 0.7 was applied after performing logistic transformation of the raw

510 DeepBind scores (39).

512 In the second part of the benchmarking impact of datasets was assessed on model building quality

513 where models were built using different datasets and various comabinations of test and train

514 datasets were analysed. Besides RBPSpot, only two tool, iDeepE and DeepCLIP, had provision to

515 build models from user provided datasets. Remaining tools have fixed models with which they

516 work and don't provide the provision to build models from user provided data. Therefore, they

517 could not be included in this part of benchmarking. Thus, ror this part, the datasets used by

518 RBPSpot (RBPSpot dataset), iDeepE, and DeepCLIP (Graphprot dataset) were used. Four

519 differenet combinations of train and test datasets (RBPSpot train and RBPSpot test, RBPSpot train

520 and Graphprot test, Graphprot train and RBPSpot test and Graphprot train and Graphprot test) were

521 used for the benchmarking to evaluate the impact of datasets on the performance of these 522 algorithms.

\section{Comparison with experimentally reported motifs}

525 A total of 29 RBPs from RNAcompete study were found overlapping with our set of 131 RBPs.

526 Their IUPAC motifs were downloaded from RNAcompete web portal. For these 29 RBPs a total of

52744 motifs were reported. Out of these 44 motifs, 35 motifs had a length of 7 bases, eight motifs had

528 a length of 6 bases, and one motif had a length of 5 bases. Four motifs out of 44, were discarded

529 due to more than 3 variable positions in a length of 7 bases. Therefore, in the final analysis a total of

53040 motifs representing 26 RBPs, were present. These motifs were scanned in the similar manner as 
531 was done with the search for motifs identified by RBPSpot approach in order to maintain an

532 unbiased motif search approach. Random data sets to evaluate the random chance observations were

533 generated from the transcriptome data using the length exactly similar to the ones from the cross-

534 linking peak data. The similar above mentioned allowed mismatches based motif searching criteria

535 was used here also to scan the random datasets for motif occurrence in them. Binomial test was

536 applied to find the significance of these motifs in the cross-linking data. Other than RNAcompete

537 motif, experimentally validated motifs were also considered from CISBP-RNA Database. A total of

53831 RBPs from this dataset were found overlapping with RBPSpot data. Out of these 31 RBPs, 24

539 RBPs were reported from RNACompete study only, two RBPs were reported through SELEX and

540 yeast three-hybrid screening whereas five RBPs were reported from RNAcompete and SELEX/RIP-

541 Chip. These motifs were also searched in the similar manner.

543 Application of RBPSpot across SARS-CoV2 genome

544 To identify the binding sites of RBPs across SARS-Cov2 genome, we downloaded its genome from 545 NCBI (accession number NC_045512).

\section{Results and Discussion}

\section{Reads data collection, filtering, and pre-processing}

550 CLIP-seq peak data from various sources were collected for 137 RBPs from starBase 2.0, also

551 know as ENCORI (Supplementary Data 1 Sheet 1). All the data were collected in the form of co-

552 ordinates. These data were from multiple types of CLIP-seq experimental techniques i.e. CLASH, 553 dCLIP, eCLIP, FLASH-CLIP-seq, HITS-CLIP, iCLIP, PAR-CLIP, sCLIP-seq, and uvCLAP. The 554 peak data varied from 234 ( PAPD5) to 9,84,503 (U2AF2) peaks. Initially, six RBPs' data were 
555 discarded due to insufficient peak data availability. Here we considered only those RBPs which

556 were having >500 unique binding peaks available. These six RBPs viz. PAPD5 (234), EIF3B (298),

557 EIF3A (371), EIF3G (398), EIF3D (399), and PUM1 (473) had lesser number of initial peaks

558 available. Remaining data for 131 RBPs were having a total number of 2,11,23,594 unique peaks.

559 To further filter this data we discarded those sequences which were having a length $<5$ nucleotides

560 or extreme length sequences (>300 basepairs). With this all, a total of 1,87,14,999 peaks were

561 available for the study, varying from EIF4A1 $(1,175)$ to AGO1-4 $(9,41,224)$. Initial co-ordinate data

562 were extracted into sequences from genome. Initial and final data are given in (Supplementary Data

5631 Sheet 2).

Most of the RBP binding sites display a prime binding motif covering majority and along with co-occuring motifs

As discussed in the introduction section, most of the available tools for identifying the RBP bindings sites across the RNAs require either prior information available traditional motif finding approaches like MEME, TOMTOM or HOMER. The application of traditional motif discovery tools may not be much information in case of RBPs which have been reported to be sparse, short, and poorly conserved. Further to this, such motif discovery approaches expect user defined motif

572 length instead of naturally capturing the motif. In general, if such motifs are not considered with 573 proper context they may lead towards false discoveries. Here, we have used the initial deep574 sequencing data to find the most frequently occurring k-mers (seeds) to make it an initial step for 575 motif finding. To find a naturally occurring most frequent k-mers, search was started with k=6 with 576 two mismatches. Reason behind this was that some of the previously reported motifs for RBPs 577 were either very sparse or as small as 4 bases long only. Therefore, a k-mer with six bases and with 578 two mismatches would fetch all possible 6-mer spectrum which would agree with each other with two mismatches (relatives to the main k-mer) while also meeting the lowest bound of such motifs. 
This defined the 6-mer groups within two mismatches. Since a large number of 6-mers spectrum is created whose search with two mismatches across the sequences becomes a computationally intensive and time consuming step, a FM-Indexing and Burrows Wheeler Transformation (BWT) based inexact search step was applied. Since, parallelism through multiprocessing was also implemented, the search becomes more faster with available cores of CPUs.

4,096 possible combinations of 6-mers were individually searched in the peak data for every RBP.

To select the most abundant 6-mers, the first criteria was its occurrence across at least $70 \%$ of the evaluated for their significance occurrence at $p$-value $<=0.01$ using binomial test. Many most frequently occurring 6-mers were found whose numbers varied for RBPs (from RBM39 (3) to ELAVL1(17)). The found significant spots for 6-mers for any given type worked as the seed which were subjected to bi-directional expansion. This expansion step every time evalauted the similarity between the expanded region and checked for minimum similarity cut-off of $70 \%$ across the considered seed regions which were expanding. The 6-mer seeds were expanded at every found position until they were satisfying both the criteria. The final step resulted into the most frequently occurring elongated k-mers with maximum possible elongation with both criteria met. After elongation, the best scoring expanded k-mer family for each RBP was considered as the primary motif in the RNA sequences interacting with the given RBP. It was found that at least one such primary motif existed for all the RBPs considered in this study, barring four RBPs. These primary motifs were occurring in at least in $70 \%$ of the data with high significance. The size of primary

601 motifs varied from 6 bases to 10 bases for different RBPs. The most abundant motif was based on 602 UCUGCAG for ALKBH5 (92.27\%), where as the least abundant motif was based on CCUGGAGG 603 for SLBP protein (Supplementary Data 1 Sheet 3). 
605 There were four different RBPs viz. FXR1, SND1, ILF3, and U2AF1 which did not have any single

606 seed k-mer occurring in at least $70 \%$ of the data. This suggested the possibility for multiple motifs

607 working in mutually exclusive manner. It was found that two different motif groups for these four

608 RBPs were working almost in mutually exclusiveness manner with small fractions of overlaps in

609 their instances. The overlap levels between these two motif groups' instances were: FXR1 (7.8\%),

610 SND1 (6.8\%), ILF3(8.25\%) and U2AF1 (9.5\%) instances (Supplementary Data 1 Sheet 4).

611 However, for these cases the found 6-mers could not be expanded further as at least $10 \%$ of the data

612 was lost due to this.

614 This way, the most significant motifs present in the cross-linking data of all these RBPs were

615 discovered which could act as anchor in contextual form. It was interesting to observe that the

616 identified motifs could be clustered into various groups based on their similarity. For every RBP, the

617 motifs obtained from their respective sequences were used to develop their position weight matrices

618 and logos which were compared with each other for similarity based clustering. This resulted into

61928 clusters of RBPs where RBPs belonging to same cluster shared good level of similarity for their

620 prime motifs (Supplementary Figure 1). Such display of grouping among RBPs is reflection of

621 unity in diversity phenomenon as well as strongly suggest that how much of importance contextual

622 factors could be for RBP-RNA interactions that despite of sharing similarity in their main motifs the

623 binding appeared highly contextual. This also transpires from the study done on the flanking

624 regions of these main motifs for the RBPs belonging to the same cluster. The di-nucleotide,

625 pentamers and heptamers based information content strongly varied among themselves for many

626 cases. The upcoming sections will present some related information on this. 
628 When these motifs were mapped back to the genome in order to derive the contextual information,

629 several of them hinted for coexistence of secondary supporting motifs for any given RBP. Such

630 cases were studied further for co-occurrence of motifs where the most dominant motif would be

631 supported by some other predominant secondary motif. All those sequences where the dominant

632 motif existed were also searched for the supporting secondary motifs. Obtained co-occurring motif

633 pairs were further evaluated to measure the similarity between them using Jaccard similarity index

634 based approach. The method utilizes the position weight matrices of co-occurring motifs for

635 alignment considering relative shifts to recognize similarity between two motifs (40). All co-

636 occurring motif pairs possessed similarity score $<0.2$ ensuring different motif partners being

637 evaluated instead of same motif repeating itself. Sequence regions where the motifs co-occurred

638 displayed high statistical significance of co-occurrence rate for the motif pairs for any given

639 distance(p-value $<<0.05$; KS-test ). For all RBP models, big difference was observed for the

640 distribution of co-occurring motif pairs when compared to the random sequence regions, strongly

641 supporting the existence of co-occurrence of motifs in RBP binding models of RNAs. Figure 2

642 illustrates some of these cases. In this way, 178 statistically significant co-occurring motifs pairs out

643 of 297 motif pairs for 127 RBPs were obtained, strongly suggesting again that context holds

644 importance in RBP-RNA interactions. Co-occuring motif details for the RBPs is given in the

645 Supplementary data 1 Sheet 8 . Further these motifs were also checked for frequency ratio $>1$ as

646 discussed in method section. All 178 statistically significant co-occurring motif pairs were found to

647 have frequency ratio (FR) $>1$. These co-occuring motifs were analyzed for the region flanking 75

648 bases from both sides of the prime motif. The reasons for considering this region becomes more

649 clear in the following next section.

651 The motifs reported in the present study were compared with the experimentally reported motifs.

652 Most of the motifs found in this study matched with the experimentally reported motifs. However, it 
653 was also observed that several of these experimentally reported motifs were not the prime motif

654 reported here but matched to other lower ranked motifs which either co-occured with the prime

655 motifs or were exclusively present, covering comparatively lesser amount of CLIP-seq data than the

656 prime motifs reported in the present study. Their occurrence in the cross-linking data varied from

$65734.07 \%$ to $81.32 \%$ while the prime motifs reported in this study mostly covered at least $70 \%$ of

658 CLIP-seq data. Figure 3 provides a snapshot of the comparison between experimentally reported

659 motifs with motifs identified in the present study.

661 Consideration of expression data for targets helps in building more realistic data-sets

662 The discovered motifs above work as a point to zero upon to consider the potential significant

663 interaction spots in the RNA. However, such motifs alone can't hold much higher stake than that as

664 they may appear in the non-binding regions also, though found statistically significant for the

665 binding regions. Evaluation of their context for their functional role thus becomes essential. In this

666 regard, 75 bases from both the flanking regions were considered where the motif region worked as

667 the anchor. Previously, it has been found that $\sim 75$ bases of flanking regions around the potential

668 interaction sites in RNAs capture the local environment for structural and contributory information

669 effectively (20). Also, RBPs which interact with the RNA through multiple domains use multiple

670 interaction sites which are usually concentrated around a local region instead of being long

671 distanced interaction spots. Thus, uniform length sequences with flanking regions were obtained for

672 every individual RBPs which varied for different RBPs depending upon the length of their anchor

673 motifs. This also led to the construction of positive and negative instances datasets, simultaneously.

674 The number of positive instances differed for the RBPs depending upon their available cross-

675 linking sequencing data, ranging from 1,309 (EIF4A1) to 8,48,680 instances (AGO1-4). This

676 covered a total of 19,547 genes experimentally confirmed as targets of these RBPs. Total number of 
677 instances was greater than total number of peak data for most of the RBPs due to multiple

678 occurrence of motifs on a single sequence.

680 Identifying suitable negative dataset candidates becomes a more crucial task. And it is where most

681 of the previously developed tools have gone too soft and mostly ended up selecting random

682 sequences, which actually does not help to divulge more information. As transpires from above

683 discussions and results, there are many spots across the transcriptomes which posses sequences

684 similar to the interaction motifs but they yet not interact. In usual, chances of finding shorter motif

685 themselves is higher in the random data. In such scenario, considering random sequences really

686 does not add significantly to the purpose of discrimination and does not answer the question raised

687 above. In order to build a better negative dataset, it is better to pick those candidates as negative

688 instances where the region similar to the main motif is present and creates a strong confusion

689 matrices to build a more natural and robust model. Therefore, to create the negative set for RBPs

690 two different kind of strategies were used. In the first strategy we used RNA-seq data for the same

691 condition for which we had the cross-linking data available for the given RBP. Those RNAs were

692 selected which were expressing themselves in the same condition but did not bind to the considered

693 RBP and did not reflect in the CLIP-seq data. They were searched for the prime motifs of the RBP

694 similar to the positive data cases and in similar manner 75 bases flanks were considered along with

695 capturing the contextual information with more discrimination power. For the RBPs for which the

696 negative datasets were created using this strategy are called Set A RBP datasets throughout this

697 study. This way, the negative datasets for 74 RBPs were created (Supplementary Data 1 Sheet 5).

699 In the second strategy, the negative datasets were created for those RBPs which did not have similar 700 condition RNA-seq data available for the considered CLIP-seq conditions. In such scenario, 
701 therefore, here those RNAs were considered which exhibited binding to their respective RBPs but

702 they also had the motifs on other positions which did reflect in the CLIP-seq data and were also far

703 away from such cross-linking regions. The logic behind is that such RNA sequences whose some

704 regions exhibited binding to RBPs in CLIP-seq data make clear positive instances out of these

705 regions as well as hold a simultaneous evidence that these RNAs were expressed in the given

706 condition. Regions which display the interaction motif in these expressed RNAs but don't bind to

707 the RBPs become an apt case for negative instance consideration with high potential for contextual

708 information unlike the usual random sequences. This particular set of negative dataset instances

709 were called Set B. In this way, the Set B negative dataset were created for the remaining 57 RBPs

710 (Supplementary Data 1 Sheet 6)). Rest of the analysis were same on both the sets of RBPs. This all

711 also reinforces the view that any successful RBP-RNA interaction discovery approach can not be

712 founded solely upon the motifs consideration but needs correctly designed context information

713 extraction approach also which can be provided only after a better a negative instances

714 consideration.

716 Contextual information surrounding the anchored motif is critical for RBP binding sites

717 recognition

718 Motif discovery and anchoring helped in selecting the more appropriate positive and negative

719 instances from which contextual information and features might be derived. The contextual

720 information came in the form of other co-occurring motifs, sequence specific information, position

721 specific information, and structural/shape information which could exhibit sharp discrimination

722 between negative and positive instances. Contextual information were derived from the features

723 based on four major properties: (1) 7-mers frequency probability for each position, (2) 5-mers

724 frequency probability for each position, (3) di-nucleotide densities in the region, and (3) Structural

725 triplet frequency covering 27 combinations of structure triplets arising from the dot-bracket 
structural representation from RNAfold predicted RNA structures. Consideration of heptamer was

for picking up any further sequence specific signals in the flanking region, where similar approach

728 of inexact search was applied with at least $70 \%$ similarity match as was done for the prime motifs’

729 6-mer seeds. Pentamers application was motivated from the recent findings which reported that

730 pentamers capture the DNA shape very accurately (24). The nucleic acids shape has been found

731 critical in the interactions with regulatory proteins which scan these shapes for their stationing. So

732 far, this approach has been applied on DNA but hardly on RNAs. The DeepBind work had

733 observed about the importance of using such kind of information which could be beneficial in

734 future developments for the tools reporting RBP-RNA interactions (14). The dinucleotide densities

735 have been found to be highly useful in indirectly evaluating the RNA structure and accessibility

736 (20,21). In fact, it has been found more promising than ab-initio RNA structure prediction. $A b$ -

737 initio methods' accuracy drastically falls with the length of RNA, and they are suitable for only

738 short RNA sequences $(9,20)$. Pentamer and di-nucleotide frequencies capture better structural and

739 shape information through base stacking and neighborhood contribution. Similarly, RNA structure

740 triplet has been used widely in deriving the structural information of RNA for their propensity

741 towards interaction factors, especially for miRNA:RNA interactions (41).

743 Various features generated based on the above mentioned properties were evaluated for their

744 discrimination potential between the positive and negative instances. The most important top 100

745 features are given in supplementary data 1 sheet 9 . Among them, the features originating from the

746 dinculeotide densities appeared the most. Some pentamer and heptamer features were also present

747 among these top features. Dinucleotide density reflects the structural and accessibility properties of

748 the nucleic acids, as mentioned above. A very striking observation was also made here. Most of the

749 positive instances flanking regions displayed enrichment of CG. Approximately 69\% of RBPs

750 target regions exhibited CG among the most dominant feature for each position. Where as for rest 
751 of the RBPs had UU and UA among the most prominent features. Besides this, it was also observed

752 that RBPs which shared high similarity for their binding motifs and were clustered among the same

753 group (Supplementary Figure 1) differed substantially for this contextual information and their

754 flanking regions displayed different distribution patterns. Figure 4 presents an example of one such

755 group, RBPs belonging to AGO4 cluster (Cluster 1). As can be noted in this figure also, CG is

756 remarkably enriched for the binding site regions. Therefore, despite of having binding sites motifs

757 they differ in their binding which is influenced by context. Also, the universal prominence of CG in

758 the RBP binding regions reinforces the theory which suggests their regulatory roles in stationing

759 the binding factors and supporting the binding motifs (42). Also, they may be studied further for

760 RNA modification which are considered critical for RBP binding dynamics.

762 For 12 RBPs, pentamers were also found in the top 20 features for different positions whereas

763 heptamers were found for 10 RBPs in the top 20 features. Among top 100 features, almost in 90\%

764 cases heptamers and pentamers marked their presence. Significant difference was observed

765 between the positive and negative instances with respect to the F-score for positions which also

766 suggest that substantial amount of information is being held by the flanking regions around the

767 binding motif, which may be one of the determinant for contextual interactions between RBP and

768 RNA. A series of t-tests between the positive and negative instances for various features also

769 supported this. Biologically, heptamers and pentamers were expected to reflect any supporting co-

770 occurring motifs near the prime anchored motif. Pentamers, specifically, were considered to

771 capture the shape properties, which too have been called important in protein and nucleic acids

772 interactions, more so in cases where sequence motifs are not clear or prime $(14,24)$. A closer look

773 with these pentamers and heptamers revealed that for many RBPs binding sites, they were

774 prominent in the flanking regions where the co-occuring secondary motifs existed (Figure 2).

775 Though heptamers were found more reflective to this phenomenon. As could be expected now, 
776 these information properties from the flanking regions looked highly promising for identification of

777 a true binding site. The impact of each of these properties on discrimination capacity between true

778 binding sites and negative sites was also clear when evaluated directly on the machine learning

779 models for performance, as transpires in the following section.

DNN implementation of the RBP binding site models consistently achieved high accuracy

782 Before combining the features to build the collective models for RBP-RNA interactions, one more assessment of contributions by the above mentioned properties in discrimination was done. Classification assessment was made for each given properties separately before joining them together while using XGBoosting. This was done to get the preliminary idea about the individual contribution made by each of the contextual properties towards the accurate classification and how important they looked in the process of accurate recognition of the binding spots. For the pentamers based classification the accuracy varied from 60.23\% (U2AF2) to 82.01\% (FKBP4) for Set A RBPs with an average of 69.8\% accuracy. For heptamers it varied from 65.01\%(FXR1) to 88.72\% (FXR2) with an average accuracy of $76.49 \%$ for set A RBPs. Similarly, for set B RBPs pentamer accuracy varied from 55.6\% (DHX9) to 86\% (EIF4A1) with an average of 66.7\% accuracy. For heptamers it varied from 57.23\% (MOV10) to $97.47 \%$ (EIF4A1) with an average accuracy of $77 \%$ for Set B RBPs. For structure triplets we used different window size but none of the windows achieved more than $63.39 \%$ accuracy for any RBP, clearly supporting our above made observation that ab-initio structure prediction derived features don’t add much value due to their innate limitations. Therefore, this feature was not further taken for the final model building. Accuracy for di-nucleotide densities based classification varied from $63.04 \%$ (FMR1) at 43 window size to $88.6 \%$ (RBFOX2) at 71

798 window size with an average of $75 \%$ accuracy at different window sizes which varied from 17 to 799103 for Set A RBPs. Similarly, for set B RBPs the accuracy of di-nucleotide density based 800 classification varied from $61.46 \%$ (DHX9) at 71 window size to $90.57 \%$ (EIF4A1) at 91 window 
801 size with an average of 75.25\% accuracy (Supplementary Data 1 Sheet 5,6). The results here

802 displayed concordance with the observation made in the previous section where importance of

803 contextual dinucleotide density information based features emerged as the most important ones for

804 the binding sites detection. Figure 5(A) presents the violin plots for the accuracy distributions

805 observed for the classifications done by each of these properties for all the RBPs.

807 With this all, it was pretty evident that the selected properties and their features had strong

808 discriminatory strength, barring the RNA structural information derived through ab-initio structure

809 prediction method. All the features originating from these qualifying properties were combined

810 together to build the final models of RBP-RNA interaction targets.

812 After getting optimum window size for di-nucleotide densities, we combined these three features

813 (pentamers probabilities, heptamers probabilities and di-nucleotide densities) together to build the

814 final models. The final models were built using Xgboosting as well as DNN. The reason for

815 considering these two different approaches are that: 1) they reflect two different learning

816 approaches: Shallow and Deep, 2) They complement each other as Xgboost works good for the

817 cases with comparatively lower training data while DNN performance is good where learning data

818 is higher, 3) Both the approaches work very good for conditions where the dimensions are high, as

819 was with this study.

821 Combining of the features based on above mentioned properties was done in a gradual manner in 822 order to see the additive effect of them on the classification performance. As it is apparent from

823 Figure 5(B), which showcases the DNN classifier's performance for five RBPs, the performance of 824 the classifiers kept increasing on the addition of more features, where consistency also increased as 
825 can be seen through the band width of the plots for the five RBPs. Here also, the dinucleotides

826 based contextual features emerged most critical as the biggest leap in the performance was noted

827 when it joined the heptamers and pentamers based features. Any pair of these three properties

828 features gave almost similar performance, but sharpest rise was observed in the performance when

829 contextual dinucleotide information based features were added to the pentameric and heptameric

830 features.

832 After combining the features we had 1,198 (ZNF184) to 2,544 (EIF4A3, EIF4G1,

833 EWSR1,HNRNPD, HNRNPL, KHDRBS3, NOP58 etc.) features for individual RBPs. The feature

834 numbers varied due to different sized best performing dinucleotide densities windows. These

835 features were used in Xgboost machine learning where the average accuracy of 85.07\% (Avg. AUC:

836 85.06\%, Avg F1-Score: 84.64\% Avg MCC:79.26) was obtained and where the values varied from

837 79.19\% (FXR2, AUC: 79.19\%, F1-Score: 78.58\% MCC:66.38) to 90.81\% (RBM47, AUC: 90.80\%,

838 F1-Score:90.49 \% MCC:83.17)) for Set A RBPs. It was found that the average accuracy of $84.08 \%$

839 (Avg. AUC: 84.07\%, Avg F1-Score:82.66 \% Avg MCC: 69.07) was obtained for Set B RBPs, where

840 accuracy values varied from 66.34\% (MOV10, AUC: 66.34\%, F1-Score: $64.74 \%$ MCC:42.58 ) to

841 96.78\% (EIF4A1,AUC: 96.48\%, F1-Score: 96.40\% MCC: 92.37). The same set of the combined

842 features was also used in the DNN implementation. DNN works better with higher dimensions and

843 instances to learn from. In the input layer combined features were used where as two hidden layers

844 gave best performance and the number of nodes per hidden layer varied from 700 to 1,300. Details

845 of implementation are already given in the methods section. DNN achieved an average accuracy of

846 92.25\% (Avg. AUC: 92.64\%, Avg F1-Score: 91.97\%, MCC:84.52\% ) for Set A RBPs which was

847 much higher than XGBoost. Whereas for Set B RBPs an average of 83.47\% (Avg. AUC: 89.61\%,

848 Avg F1-Score: 83.18\%, Avg MCC:67.34\% ) accuracy was achieved by the DNN models, which was 
849 slightly lower than XGBoost. Complete performance details can be found elsewhere

850 (Supplementary Data 1 Sheet 5,6).

852 In general, it was apparent that DNN approach was sensitive towards the volume of training

853 instances as it was found performing better where number of instances were higher. But the biggest

854 impact on performance was observed was for the granularity of dataset creation. Performance of

855 DNN was specially more marked here, as can be seen from its performance plot on Set A datasets.

856 On Set A, the DNN models performance hardly touched below 90\% accuracy. Even XGBoost’s

857 performance was better with Set A when compared to Set B. It needs to be recalled that Set B was

858 made for those RBPs for which the RNA-seq data was not available for the considered CLIP-seq

859 conditions. In such scenario, those RNA were considered to generate the negative instances whose

860 some regions were present in the CLIP-seq data suggesting their expression. From the same RNA,

861 those regions were selected which were having the prime motifs but yet not binding to the RBP and

862 not reflected in the CLIP-seq data and were distant from such binding regions. While Set A negative

863 instances were clearly those regions which were expressed during the CLIP-seq experimental

864 condition and possessed the prime motif but no region of the RNA itself bound to the RBP. Thus,

865 though the over all performance with Set B was still good and better than the datasets used by the

866 compared tools as transpires in the next section, it same time reflects that how important it is to

867 have a refined data-set like Set A. This is possible that some instances covered as negative instances

868 in Set B could be contributing to the RBP-RNA interactions or could not be captured in the CLIP-

869 seq experiments. Yet, as transpires from the various performance metrics plots across various RBPs

870 given in Figure 6 and AUC/ROC plots given in Figure 7, the developed approach in this study,

871 named as RBPSpot, showcases a consistently high and reliable performance for a large number of

872 RBPs. It also provides the largest number of models for RBPs binding developed from CLIP-seq

873 data to this date. 


\section{Comparative benchmarking: RBPSpot consistently outperforms all the compared tools}

876 A very comprehensive benchmarking study was performed where RBPSpot was compared with five

877 different tools, representing different approaches of RBP RNA interaction detection: RBPmap

878 (probabilistic approach), beRBP (Random Forest machine learning bases claiming highest accuracy

879 in its category), DeepBind (the first deep-learning based approach), iDeepE and DeepCLIP

880 (representing some very recent and more complex deep-learning based tools). Besides this, the

881 benchmarking has also considered three different datasets as this work also presents a new dataset

882 while underlining the importance of better datasets in creating better models as well as to carry out

883 a totally unbiased assessment of performance of these tools on different datasets.

885 Thus, the first dataset considered in the benchmarking study was derived from the RBPSpot dataset.

886 Only those RBPs were considered for comparison for which at least one tool had model built,

887 besides RBPSpot itself. This way comparison was done for 52 RBPs. The second dataset considered

888 was the one evolved during development of Graphprot software which has been used largely by

889 various other datasets for model building and performance benchmarking purposes. The third

890 dataset used in this benchmarking study was the one used by beRBP software which too has been

891 used by many other tools for the same purpose. Details about these datasets have already been

892 discussed above and in the methods sections.

894 All these six software were tested across all these three datasets and RBPSpot outperformed all of 895 them across all the datasets, and for all the performance metrics considered (Figure 8). Figure 8 896 gives a detailed view of the data analysis of this benchmarking across the three datasets studied for 897 all these software. RBPSpot scored the average accuracy of $88.43 \%$ and the average MCC value of 8980.77 on RBPSpot dataset, the average accuracy of $91.63 \%$ and the average MCC value of 0.83 on 
899 Graphprot dataset, and the average accuracy of $88.9 \%$ and the average MCC value of 0.74 on

900 beRBP dataset. Among all the considered performance metrics, MCC stands as the most important

901 one as it gives high score only when a software scores high on all the four performance parameters

902 (true positive, false positive, true negative, false negative). A good MCC score signifies the

903 robustness of the model and its performance consistency. RBPSpot emerged as the most robust

904 algorithm among all these compared software with very high consistency of performance. As it is

905 visible from the score distribution for all the metrics, RBPSpot also exhibited least dispersion of

906 scores for all the studies RBPs and for all the three datasets, confirming the precise performance

907 achieved by RBPSpot compared to other tools.

909 After RBPSpot, the best performance was observed for the complex deep-learning based software

910 iDeepE and DeepCLIP. On RBPSpot dataset, iDeepE performed better than DeepCLIP, but for other

911 two datasets they attained almost similar metrics scores for performance. Undeniably, they emerged

912 far superior than their deep-learning predecessor, DeepBind, and other compared tools. They even

913 displayed much smaller dispersion of their scores than other compared tools. However, RBPSpot's

914 performance points out that more appropriate features may be learned through training on

915 biologically relevant properties to derive better discrimination power using machine learning 916 approach, which can be amalgamated with Deep Neural Nets with much lesser complexity and

917 superior performance than applying complex deep-learning layers to automate feature extraction.

918 The observations made in the introduction part of this work appeared true in this study that such

919 complex deep-learning approaches score good on unstructured data where clear features

920 identification and extraction is difficult to be done by expert and automation is required for feature

921 extraction. The problems where features are identifiable and can be structured, simpler machine

922 learning models may outperform the complex deep-learning approaches. 
924 The above mentioned benchmarking was done for all the tools while keeping their original training

925 dataset and models for RBPs. Most of the existing tools don’t provide the option to build user

926 specified models of RBPs using their algorithms but come with their own pre-built models. This

927 limits the scope to test the algorithms with different combinations of datasets. Fortunately, the two

928 best performing tools after RBPSpot, iDeepE and DeepCLIP, provided this scope where the users

929 may build their new models with their own datasets. Also, since these two tools performance were

930 next to RBPSpot, they stood as a natural choice to study the performance impact with datasets

931 variations. Both iDeepE and DeepCLIP have implemented Graphprot dataset for their original

932 model building. For this part of benchmarking study the training and testing datasets of RBPSpot,

933 iDeepE, and DeepCLIP were swapped and studied for four different combinations of training and

934 testing datasets: RBPSpot training and testing datasets, RBPSpot training and Graphprot testing

935 dataset, Graphprot training and testing datasets, Graphprot training and RBPSpot testing dataset.

937 Figure 9 presents the results for this part of benchmarking where RBP models were rebuilt and

938 tested using the four different combinations of training and testing datasets. RBPSpot outperformed

939 the remaining two software, iDeepE and DeepCLIP for all the combinations of datasets, for all the

940 considered performance metrics. Like the previous benchmarking study, here also RBPSpot scored

941 the highest among all the software for all the combinations of datasets with a remarkable

942 consistency. As transpires from the kernal density plots in Figure 9, RBPSpot maintained its least

943 variability and dispersion of performance scores and continued to display it strong balance in

944 detecting the positive and negative instances with high and similar level of precision. This was

945 reflected by high scoring on all the four parameters of performance resulting into consistently

946 highest MCC values, which confirmed the robustness of the algorithm. Also, it was observed that

947 performance of all the compared software was better when RBPSpot dataset was used for training.

948 The original implementation of iDeepE and DeepCLIP have used Graphprot dataset. Both these 
949 software performed better when their original dataset for model building was replaced by RBPSpot

950 training dataset, underscoring better and more realistic composition of RBPSpot dataset.

951

952 The benchmarking done here stands among one of the most comprehensive ones. It looked into

953 various aspects of performances and has involved a large number of RBPs for comparison as well

954 as evaluated the role of datasets in performance. RBPSpot consistently scored high across all the

955 comparative tests and clearly outperformed the compared tools. The full details and data for the

956 benchmarking studies are given in supplementary Data 1 Sheet 10-15.

958 Structural and molecular dynamics analysis supports the RBP binding site models

959 Depending upon the availability of complete experimentally validated 3D structures in PDB

960 database, structures for 13 RBPs ( IGF2BP1, DIS3L2, CNBP, SRSF3, FKBP4, KHDRBS1,

961 LIN28A, CAPRIN2, DICER1, GTF2F1, HNRNPC, CPSF6 and AGO2) were selected for the

962 structural interaction analysis for the identified binding sites (43). In order to examine

963 conformational variations of the RBPs within the hydrated controlled environment, the root-mean-

964 square deviation (RMSD) of the atomic positions of RNAs containing motif with respect to RBP

965 backbone were calculated and compared with the RNA complexes without the prime motif. In

966 comparative analysis of RMSD measures these RBPs complexes were considered with three

967 different RNA sequences for each RBP. These sequences were randomly selected from positive

968 datasets having 75 bases flanking regions. To analyze the structural behavior of RBPs and their

969 complexes, 20 ns simulation job was performed. For this purpose, selected RBPs and complexes

970 were immersed in the cubic boxes of varying dimensions based on the system size. Prior to the

971 energy minimization process, different charged molecules like $\mathrm{NA}^{+}$or $\mathrm{Cl}^{-}$were added to neutralize

972 the system (44). 
974 Once the simulation was finished, the last step was to analyze the simulation result in term of

975 RMSD plot during the course of simulation for 20ns. RMS module in GROMACS was executed

976 while choosing "Backbone" for least-squares fitting and "RNA_Heavy" for the RMSD calculation.

977 By doing so, the overall rotation and translation of the protein was removed via fitting and the

978 RMSD reported about how much the RNA position varied relative to the protein. This is considered

979 as a good indicator of how well the binding pose was preserved during the simulation. Comparative

980 analysis of RMSD trajectories of 13 different RBPs-RNA complexes with three replicates each for

981 the two conditions clearly suggested that the presence of the identified prime motifs was giving

982 stability to the RBP-RNA complexes (Figure 10).

984 For example, in case of AGO2, on comparative analysis of RMSD value of the AGO2-RNA first 985 sequence complex with the prime motif, the value ranged from $0.1 \mathrm{~nm}$ to $0.7 \mathrm{~nm}$ and got stabilized 986 at $0.5 \mathrm{~nm}$ whereas RMSD values for the complex without the motif ranged from $0.1 \mathrm{~nm}$ to $1.7 \mathrm{~nm}$ 987 and got stabilized at $1.5 \mathrm{~nm}$, which was less stable. Similarly the second pair with motif had RMSD 988 ranging from $0.1 \mathrm{~nm}$ to $1.7 \mathrm{~nm}$ which got stabilized at $0.6 \mathrm{~nm}$, whereas the same pair without the 989 prime motif ranged had RMSD ranging from $0.3 \mathrm{~nm}$ to $1.4 \mathrm{~nm}$ and got stabilized at $1.4 \mathrm{~nm}$. For the 990 third pair, the AGO2-RNA complex of the third sequence with the prime motif showed deviation 991 from $0.1 \mathrm{~nm}$ to $1.0 \mathrm{~nm}$ and got settled at $0.7 \mathrm{~nm}$ whereas the same sequence without the prime motif 992 showed deviation from $0.0 \mathrm{~nm}$ to $2.0 \mathrm{~nm}$ and settled at $1.4 \mathrm{~nm}$. In all the three cases of AGO-RNA 993 complexes, the sequence with the prime motif was found to be more stable when compared to the 994 one without the motif in the dynamic environment. Similar pattern was observed for all the 13 RBP 995 and their triplicate pairs. Details can be found in Table 1. 
997 In the nutshell, the structural molecular dynamics study supported the identified binding spots for

998 the RBP where it was clearly evident that the identified binding motif provided structural stability to

999 the considered RBP-RNA complexes.

1000

1001 Application: SARS-Cov2 genome was found to host RBP binding sites

1002 Most of the deadly viruses are RNA viruses which exploit the host proteins to replicate, spread and 1003 survive. The best living example is nSARS-CoV-2. The emergence of the novel human corona-virus 1004 SARS-CoV-2 in Wuhan, China has caused a pandemic of respiratory disease (Covid19). The big 1005 scientific concern is that to this date very scarce and uncertain molecular information is available 1006 about the Covid19 patient's molecular system as not much high-throughput studies have been 1007 carried out so far. There is almost absolutely no information on the host RBPs response during 1008 Covid19 infection despite of the fact that all such virus essentially require host RBPs to survive and 1009 replicate And RBP-RNA interaction studies hold prime importance in this regard also.

1011 Therefore, we scanned the SARS-CoV-2 genome through RBPSpot to find the binding sites for 1012 RBPs which could have therapeutic value. Interestingly, out of 131 different model we found 22 1013 different binding sites for 7 different RBPs (AIFM1 (2), BUD13 (3), CELF2 (4), RBM6 (3), UPF1 1014 (2), TARBP2 (4) and KHSRP (4)) (Figure 11). Among these, AIFM1 interaction with viral 1015 polymerases in influenza virus infected cells is well studied (45). These all binding sites were found 1016 on anti-sense strand of the genome whose importance is for viral replication. During the infection, 1017 majority of immunoprecipitated RNA of Coronavirus were found originating from the anti-sense 1018 strand (46). Therefore, there is a possibility that these RBPs are helping in it's transcription by 1019 binding to it’s negative strand. To check the stability of these RBPs with their binding site we also 1020 performed MD simulations study on two different sequence forms for each identified binding site 
1021 (One with the binding site and another without it). Prior to this, we obtained complete 3D structures

1022 for AIFM1 and UPF1 from PDB and modeled the remaining five RBPs through homology 1023 modeling due to lack of complete defined structures for them. After modeling we evaluated the built 1024 3D structure models using SAVES v6.0 (structure Activity validation server). Five RBPs PDB 1025 structures namely AIFM1, BUD13, CELF2, TARBP2 and UPF1 passed through verification filter 1026 like PROCHECK and WHATCHECK except KHSRP and RBM6. When we analyzed the model 1027 structure for KHSRP and RBM6 with both program it gives 80.9\% and 83.5\% residues in allowed 1028 regions in the Ramachandran plot but for good quality model, over 90\% residues are expected in the 1029 most favored region and lack of loop filtering causing side-chain packing inaccuracies. 1030 Subsequently, on analyzing the RMSD graph (Supplementary Figure 2) for all the seven RBPs it 1031 was found that that five out of seven RBP-RNA complexes were stable with prime motif compared 1032 to the RBP-RNA complexes counterpart without the main motif. This part of the study was done 1033 just to showcase the application of the developed approach. The finding made in this section may be 1034 used for further study for Covid research groups.

1038 A living system is a continuous outcome of the regulatory setup working for that system in the 1039 background. RNA binding proteins define one such critical regulatory component of the system 1040 which is present at almost every post-transcriptional regulatory event but about which our 1041 understanding is still nascent and evolving. How they select their targets and carry out interactions 1042 in functional manner is largely ambiguous. With the advent of high-throughput techniques like 1043 CLIP-seq and interactome capture, the information on genes recognized as RBPs and their 1044 interactions are growing continuously. Such high-throughput data on interactions are very valuable 
1045 resources to construct the interaction models. The present study used the same from CLIP-seq

1046 experiments. However, there are several other critical factors involved which are required to be 1047 build these interactions models with high accuracy. This involves proper negative data-sets 1048 screening, appropriate motif discovery strategy, and contextual information derivation. All of them 1049 are interconnected with each other and success of any such RBP binding site discovery tool depends 1050 highly on this. Without proper datasets, correct binding specific motif candidates are hard to be 1051 found. The motif finding step itself needs to consider the sparse nature of RBP binding sites and 1052 need to anchor correctly so that correct surrounding could be recognized to provide the contextual 1053 information. Otherwise, such motifs occur frequently even in the non-binding regions, and wrong 1054 context may easily compromise the accuracy. When all these information are applied through 1055 effective machine learning algorithms, consistently high level accuracy is achievable. It was 1056 comprehensively and comparatively benchmarked against some recent tools where it outperformed 1057 them consistently across a wide number of datasets and RBPs. It also showcased that when a DNN 1058 is trained properly on suitable properties with appropriate biological insights, the developed system 1059 could easily outperform much complex deep-learning based approaches where such learning is done 1060 through automated feature extraction process using complex layers like CNN and LSTM etc. Such 1061 complex deep learning approach may be suitable for unstructure data where features could not be 1062 identified easily. However, when features are identifiable and structured, simpler machine learning 1063 approaches can outperform them easily. The developed approach in this study, RBPSpot, can 1064 identify the binding sites of existing RBPs in human system as well as it becomes one of few tools 1065 where users can put their own data and raise their own models for any species and any RBP. The 1066 software is freely available as a webserver as well as as an standalone program.

1068 From here, we visualize that incorporation of spatio-temporal and other interactome network 1069 information for RBPs as the another dimension to explore to further improve our understanding on 
1070 RBP-RNA interactions. This is something which still remains largely unaddressed. Some 1071 encouraging recent developments have happened $(47,48)$ which promise that incorporation of back1072 end network and interaction information on RBP RNA interactions could add more value towards 1073 recognition of functional and dynamic nature of RBP RNA interactions which could further boost 1074 interaction spot identification process. Also, the findings made here from the contextual information 1075 like CG enrichment in the flanking regions must be explored further for their functional roles 1076 associated with such binding sites. RNA modifications on CG and likewise other important 1077 contextually important factors found in this study may further provide reasoning for spatio-temporal 1078 nature of these interactions which would mark another level of development in our understanding 1079 towards RBP RNA interactions and regulation.

\section{Declarations}

\section{Availability of data and materials}

1083 All the secondary data used in this study were publicly available and their due references and 1084 sources have been provided. All data and information generated/used, methodology related details 1085 etc have also been been made available in the supplementary data files provided along with and also 1086 made available through the related open access server at https://scbb.ihbt.res.in/RBPSpot/. The 1087 software has also been made available at Github at:https://github.com/SCBB-LAB/RBPSpot

\section{Competing interests}

1090 The authors declare that they have no competing interests. 


\section{Funding}

1093 RS is thankful to Department of Biotechnology, Govt. of India for supporting this study through 1094 grant in Big Data analysis[Grant number: BT/PR16331/BID 17/589/2016 (GAP-0228)] to RS.

\section{Authors' contributions}

1097 NKS and SG carried out the computational part and benchmarking of the study. PK developed the 1098 FM-Index and BWT based inexact k-mer search script. AK carried out the structural analysis and 1099 molecular dynamics simulation. UKP helped in statistical analysis. RS conceptualized, designed, 1100 analyzed and supervised the entire study. NKS, SG, AK and RS wrote the MS.

\section{Acknowledgments}

1103 We are thankful to the Director, CSIR-IHBT, for his kind support. We are thankful to Dr. Indu 1104 Gangwar for her inputs for the study. We are thankful to DBT for the funding support they gave for 1105 this project. NKS is thankful to CSIR for financial support as project associateship. UKP and PK 1106 are thankful to ICAR, New Delhi for providing support in Ph.D. UKP, NKS, and PK are thankful to 1107 Academy of Scientific and Innovative Research (AcSIR).

1110 Not applicable. 
1113 Not applicable.

1114

1115 References

1116 1. Gerstberger S., Hafner M., Tuschl T. 2014. A census of human RNA-binding proteins. $1117 \quad$ Nature Reviews Genetics 15:829-845.

1118 2. Castello,A., Hentze,M.W. and Preiss,T. (2015) Metabolic Enzymes Enjoying New 1119 Partnerships as RNA-Binding Proteins. Trends Endocrinol Metab, 26, 746-757.

3. Ray,D., Kazan,H., Cook,K.B., Weirauch,M.T., Najafabadi,H.S., Li,X., Gueroussov,S., Albu,M., Zheng,H., Yang,A., et al. (2013) A compendium of RNA-binding motifs for decoding gene regulation. Nature, 499, 172-177.

4. Cook KB., Kazan H., Zuberi K., Morris Q., Hughes TR. 2011. RBPDB: a database of RNAbinding specificities. Nucleic Acids Research 39:D301-D308.

5. Khorshid M., Rodak C., Zavolan M. 2011. CLIPZ: a database and analysis environment for experimentally determined binding sites of RNA-binding proteins. Nucleic Acids Research 39:D245-252.

6. Hu,B., Yang,Y.-C.T., Huang,Y., Zhu,Y. and Lu,Z.J. (2017) POSTAR: a platform for exploring post-transcriptional regulation coordinated by RNA-binding proteins. Nucleic Acids Res, 45, D104-D114.

7. Yang,Y.-C.T., Di,C., Hu,B., Zhou,M., Liu,Y., Song,N., Li,Y., Umetsu,J. and Lu,Z.J. (2015) CLIPdb: a CLIP-seq database for protein-RNA interactions. BMC Genomics, 16, 51.

8. Kazan,H., Ray,D., Chan,E.T., Hughes,T.R. and Morris,Q. (2010) RNAcontext: A New Method for Learning the Sequence and Structure Binding Preferences of RNA-Binding Proteins. PLOS Computational Biology, 6, e1000832.

9. Gardner,P.P. and Giegerich,R. (2004) A comprehensive comparison of comparative RNA structure prediction approaches. BMC Bioinformatics, 5, 140. 
10. Paz,I., Kosti,I., Ares,M., Cline,M. and Mandel-Gutfreund,Y. (2014) RBPmap: a web server for mapping binding sites of RNA-binding proteins. Nucleic Acids Res, 42, W361-W367.

11. Weyn-Vanhentenryck,S.M. and Zhang,C. (2016) mCarts: Genome-Wide Prediction of Clustered Sequence Motifs as Binding Sites for RNA-Binding Proteins. Methods Mol Biol, 1421, 215-226.

12. Maticzka, D., Lange, S.J., Costa, F., and Backofen, R. (2014). GraphProt: modeling binding preferences of RNA-binding proteins. Genome Biol 15, R17.

13. Yu,H., Wang,J., Sheng,Q., Liu,Q. and Shyr,Y. (2019) beRBP: binding estimation for human RNA-binding proteins. Nucleic Acids Res, 47, e26.

14. Alipanahi,B., Delong,A., Weirauch,M.T. and Frey,B.J. (2015) Predicting the sequence specificities of DNA- and RNA-binding proteins by deep learning. Nature Biotechnology, 33, 831-838.

15. Pan,X. and Shen,H.-B. (2017) RNA-protein binding motifs mining with a new hybrid deep learning based cross-domain knowledge integration approach. BMC Bioinformatics, 18, 136.

16. Pan, X., and Shen, H.-B. (2018). Predicting RNA-protein binding sites and motifs through combining local and global deep convolutional neural networks. Bioinformatics 34, 34273436. BMC Genomics 19, 511.

18. Ghanbari, M., and Ohler, U. (2020). Deep neural networks for interpreting RNA-binding protein target preferences. Genome Res 30, 214-226.

19. Grønning, A.G.B., Doktor, T.K., Larsen, S.J., Petersen, U.S.S., Holm, L.L., Bruun, G.H., Hansen, M.B., Hartung, A.-M., Baumbach, J., and Andresen, B.S. (2020). DeepCLIP: 
predicting the effect of mutations on protein-RNA binding with deep learning. Nucleic Acids Res 48, 7099-7118.

20. Heikham,R. and Shankar,R. (2010) Flanking region sequence information to refine microRNA target predictions. J Biosci, 35, 105-118.

21. Černý,J., Božíková,P., Svoboda,J. and Schneider,B. (2020) A unified dinucleotide alphabet describing both RNA and DNA structures. Nucleic Acids Research, 48, 6367-6381.

22. Jankowsky,E. and Harris,M.E. (2015) Specificity and nonspecificity in RNA-protein interactions. Nature Reviews Molecular Cell Biology, 16, 533-544.

23. A,G. and M,G. (2011) The role of RNA sequence and structure in RNA--protein interactions. Journal of molecular biology, 409.

24. Zhou,T., Yang,L., Lu,Y., Dror,I., Dantas Machado,A.C., Ghane,T., Di Felice,R. and Rohs,R. (2013) DNAshape: a method for the high-throughput prediction of DNA structural features on a genomic scale. Nucleic Acids Research, 41, W56-W62.

25. Ryan M. (2021). Deep Learning with Structured Data [Book] . Manning Publications.

26. Li J-H., Liu S., Zhou H., Qu L-H., Yang J-H. 2014. starBase v2.0: decoding miRNAceRNA, miRNA-ncRNA and protein-RNA interaction networks from large-scale CLIP-Seq data. Nucleic Acids Research 42:D92-97.

27. Lorenz,R., Bernhart,S.H., Höner zu Siederdissen,C., Tafer,H., Flamm,C., Stadler,P.F. and Hofacker,I.L. (2011) ViennaRNA Package 2.0. Algorithms for Molecular Biology, 6, 26.

28. Chen,Y.-W. and Lin,C.-J. (2006) Combining SVMs with Various Feature Selection Strategies. In Guyon,I., Nikravesh,M., Gunn,S., Zadeh,L.A. (eds), Feature Extraction: Foundations and Applications, Studies in Fuzziness and Soft Computing. Springer, Berlin, Heidelberg, pp. 315-324.

29. Chicco, D., and Jurman, G. (2020). The advantages of the Matthews correlation coefficient (MCC) over F1 score and accuracy in binary classification evaluation. BMC Genomics 21, 
6.

30. Tuszynska I., Bujnicki JM. 2011. DARS-RNP and QUASI-RNP: new statistical potentials for protein-RNA docking. BMC bioinformatics 12:348.

31. Chen A-J., Paik J-H., Zhang H., Shukla SA., Mortensen R., Hu J., Ying H., Hu B., Hurt J., Farny N., Dong C., Xiao Y., Wang YA., Silver PA., Chin L., Vasudevan S., Depinho RA.

32. Berendsen HJC., van der Spoel D., van Drunen R. 1995. GROMACS: A message-passing parallel molecular dynamics implementation. Computer Physics Communications 91:43-56. 2012. STAR RNA-binding protein Quaking suppresses cancer via stabilization of specific miRNA. Genes \& Development 26:1459-1472.

33. .Duan Y., Wu C., Chowdhury S., Lee MC., Xiong G., Zhang W., Yang R., Cieplak P., Luo R., Lee T., Caldwell J., Wang J., Kollman P. 2003. A point-charge force field for molecular mechanics simulations of proteins based on condensed-phase quantum mechanical

34. Jorgensen WL., Chandrasekhar J., Madura JD., Impey RW., Klein ML. 1983. Comparison of simple potential functions for simulating liquid water. The Journal of Chemical Physics 79:926-935.

35. Hess B., Bekker H., Berendsen HJC., Fraaije JGEM. 1997. LINCS: A linear constraint solver for molecular simulations. Journal of Computational Chemistry 18:1463-1472.

36. Gunsteren WFV., Berendsen HJC. 1988. A Leap-frog Algorithm for Stochastic Dynamics. Molecular Simulation 1:173-185.

37. Vandenbon,A., Kumagai,Y., Akira,S. and Standley,D.M. (2012) A novel unbiased measure for motif co-occurrence predicts combinatorial regulation of transcription. BMC Genomics, 
38. Dassi,E., Re,A., Leo,S., Tebaldi,T., Pasini,L., Peroni,D. and Quattrone,A. (2014) AURA 2: Empowering discovery of post-transcriptional networks. Translation (Austin), 2, e27738.

39. Yuan,H., Kshirsagar,M., Zamparo,L., Lu,Y. and Leslie,C.S. (2019) BindSpace decodes transcription factor binding signals by large-scale sequence embedding. Nature Methods, 16, 858-861.

40. Vorontsov IE., Kulakovskiy IV., Makeev VJ. 2013. Jaccard index based similarity measure to compare transcription factor binding site models. Algorithms for molecular biology: AMB $8: 23$.

41. Xue,C., Li,F., He,T., Liu,G.-P., Li,Y. and Zhang,X. (2005) Classification of real and pseudo microRNA precursors using local structure-sequence features and support vector machine. BMC Bioinformatics, 6, 310.

42. Hartl,D., Krebs,A.R., Grand,R.S., Baubec,T., Isbel,L., Wirbelauer,C., Burger,L. and Schübeler,D. (2019) CG dinucleotides enhance promoter activity independent of DNA methylation. Genome Res, 29, 554-563.

43. Rose, P. W., Beran, B., Bi, C., Bluhm, W. F., Dimitropoulos, D., Goodsell, D. S., ... \& Bourne, P. E. (2010). The RCSB Protein Data Bank: redesigned web site and web services. Nucleic acids research, 39(suppl_1), D392-D401.

44. Pfeiffer, S., Fushman, D., \& Cowburn, D. (1999). Impact of $\mathrm{Cl}-$ and $\mathrm{Na}+$ ions on simulated structure and dynamics of $\beta A R K 1$ PH domain. Proteins: Structure, Function, and Bioinformatics, 35(2), 206-217.

45. Bradel-Tretheway,B., Mattiacio,J., Krasnoselsky,A., Stevenson,C., Purdy,D., Dewhurst,S. and Katze,M. (2011) Comprehensive Proteomic Analysis of Influenza Virus Polymerase Complex Reveals a Novel Association with Mitochondrial Proteins and RNA Polymerase Accessory Factors. Journal of virology, 85, 8569-81. 
46. Hackbart,M., Deng,X. and Baker,S.C. (2020) Coronavirus endoribonuclease targets viral polyuridine sequences to evade activating host sensors. PNAS, 117, 8094-8103.

47. Pradhan,U.K., Anand,P., Sharma,N.K., Kumar,P., Kumar,A., Pandey,R., Padwad,Y. and Shankar,R. (2020) Various RNA-binding proteins and their conditional networks explain miRNA biogenesis.(Under review).

48. Mukherjee,N., ～Wessels,H.-H., ～Lebedeva,S., $\quad$ Sajek,M., Ghanbari,M., $\quad$ Garzia,A., Munteanu,A., Yusuf,D., Farazi,T., Hoell,J.I., et al. (2019) Deciphering human ribonucleoprotein regulatory networks. Nucleic Acids Research, 47, 570-581.

\section{Tables}

1244 Table 1: Table for RMSD value for selected 13 RBPs complexes with and without the prime motif.

1245 The identified prime motifs were found statistically enriched in the target sequences when 1246 compared to random regions. Molecular dynamics studies with and without these motifs clearly 1247 suggested their important role in binding where they were found responsible for stable complex 1248 formation between RBP and RNA.

\begin{tabular}{|l|l|l|l|l|l|}
\hline $\begin{array}{c}\text { RBPs } \\
\text { name }\end{array}$ & $\begin{array}{c}\text { Sequence } \\
\text { name }\end{array}$ & $\begin{array}{c}\text { RMS Deviation } \\
\text { range value with } \\
\text { motif } \\
\mathbf{( n m )}\end{array}$ & $\begin{array}{c}\text { Stablized_RMSD } \\
\text { value with motif } \\
\mathbf{( n m )}\end{array}$ & $\begin{array}{c}\text { RMS Deviation } \\
\text { range value } \\
\text { without motif } \\
\mathbf{( n m )}\end{array}$ & $\begin{array}{c}\text { Stabilized RMSD } \\
\text { value without motif } \\
\text { (nm) }\end{array}$ \\
\hline AGO2 & RNA_seq1 & $0.1-0.7$ & 0.5 & $0.1-1.7$ & 1.5 \\
\hline & RNA_seq2 & $0.1-1.7$ & 0.6 & $0.3-1.4$ & 1.4 \\
\hline & RNA_seq3 & $0.1-1.0$ & 0.7 & $0.1-2.0$ & 1.4 \\
\hline & & & & & \\
\hline CAPRIN2 & RNA_seq1 & $0.1-0.3$ & 0.2 & $0.6-2.1$ & 1.9 \\
\hline & RNA_seq2 & $0.3-1.8$ & 1.3 & $0.7-2.2$ & 1.5 \\
\hline & RNA_seq3 & $0.2-2.1$ & 1.8 & $0.1-2.9$ & 2.7 \\
\hline & & & & & \\
\hline CNBP & RNA_seq1 & $0.1-1.3$ & 1.1 & $0.3-4.8$ & 4.3 \\
\hline & RNA_seq2 & $0.3-1.8$ & 0.5 & $0.3-2.5$ & 2.0 \\
\hline & RNA_seq3 & $0.1-2.7$ & 2.5 & $0.1-4.7$ & 3.4 \\
\hline & & & & & \\
\hline CPSF6 & RNA_seq1 & $0.3-1.2$ & 1.0 & $0.3-3.2$ & 3.0 \\
\hline & RNA_seq2 & $0.3-2.0$ & 1.8 & $0.3-2.8$ & 2.4 \\
\hline & RNA_seq3 & $0.1-2.9$ & 2.7 & $0.1-4.8$ & 4.1 \\
\hline
\end{tabular}




\begin{tabular}{|c|c|c|c|c|c|}
\hline \multirow[t]{3}{*}{ DICER1 } & RNA seq1 & $0.3-1.3$ & 1.0 & $0.3-3.5$ & 2.9 \\
\hline & RNA_seq2 & $0.3-1.5$ & 1.5 & $0.3-2.7$ & 2.0 \\
\hline & RNA_seq3 & $0.1-2.5$ & 2.3 & $0.1-4.8$ & 4.2 \\
\hline \multirow[t]{3}{*}{ DIS3L2 } & RNA_seq1 & $0.1-0.3$ & 0.3 & $0.3-2.5$ & 2.5 \\
\hline & RNA_seq2 & $0.3-1.8$ & 1.2 & $0.3-2.4$ & 2.0 \\
\hline & RNA_seq3 & $0.1-2.3$ & 1.8 & $0.1-4.2$ & 4.0 \\
\hline \multirow[t]{3}{*}{ FKBP4 } & RNA seq1 & $0.3-1.5$ & 1.3 & $0.3-1.2$ & 1.3 \\
\hline & RNA_seq2 & $0.1-1.7$ & 1.1 & $0.1-2.5$ & 2.2 \\
\hline & RNA_seq3 & $0.1-1.9$ & 0.5 & $0.1-2.3$ & 2.2 \\
\hline \multirow[t]{3}{*}{ GTF2F1 } & RNA seq1 & $0.3-1.3$ & 1.3 & $0.3-3.7$ & 2.5 \\
\hline & RNA_seq2 & $0.3-1.9$ & 1.7 & $0.5-2.5$ & 2.0 \\
\hline & RNA_seq3 & $0.1-2.6$ & 2.1 & $0.1-4.7$ & 4.0 \\
\hline \multirow[t]{3}{*}{ HNRNPC } & RNA seq1 & $0.1-0.5$ & 0.3 & $0.1-2.7$ & 2.3 \\
\hline & RNA_seq2 & $0.1-2.3$ & 1.8 & $0.1-2.3$ & 2.0 \\
\hline & RNA_seq3 & $0.1-2.5$ & 2.2 & $0.3-4.3$ & 4.1 \\
\hline \multirow[t]{3}{*}{ IGF2BP1 } & RNA_seq1 & $0.3-1.7$ & 0.9 & $0.3-2.6$ & 2.1 \\
\hline & RNA_seq2 & $0.3-1.5$ & 0.6 & $0.07-1.7$ & 1.3 \\
\hline & RNA_seq3 & $0.1-1.7$ & 1.5 & $0.1-2.6$ & 1.6 \\
\hline \multirow[t]{3}{*}{ KHDRBS1 } & RNA_seq1 & $0.3-1.9$ & 0.5 & $1.0-4.8$ & 3.5 \\
\hline & RNA_seq2 & $0.3-1.2$ & 1.2 & $0.3-2.1$ & 1.2 \\
\hline & RNA_seq3 & $0.3-2.5$ & 2.5 & $0.1-4.7$ & 4.2 \\
\hline \multirow[t]{3}{*}{ LIN28A } & RNA_seq1 & $0.3-1.4$ & 0.7 & $0.3-3.0$ & 2.2 \\
\hline & RNA_seq2 & $0.5-2.1$ & 1.5 & $0.5-3.0$ & 1.6 \\
\hline & RNA_seq3 & $0.1-2.2$ & 2.2 & $0.1-4.9$ & 3.1 \\
\hline \multirow[t]{3}{*}{ SRSF3 } & RNA_seq1 & $0.2-0.5$ & 0.8 & $0.5-3.7$ & 3.0 \\
\hline & RNA_seq2 & $0.3-1.2$ & 1.2 & $0.5-2.5$ & 2.1 \\
\hline & RNA_seq3 & $0.1-3.0$ & 2.5 & $1.0-4.8$ & 3.2 \\
\hline
\end{tabular}

\section{Figure legends}

1251 Figure 1: Detailed pipeline of the workflow. The image provides the brief outline of entire

1252 computation protocol implemented to develop accurate RBP RNA interaction model and identify

1253 the correct RBP binding sites across given RNA sequences. The process of model building starts

1254 from identifying prime motifs through k-mer spectrum search from the CLIP-seq regions where

1255 BWT/FM indexing based inexact search algorithm was implemented. The statistically enriched k-

1256 mers were expanded across all reporting sequences region till at least $70 \%$ similarity between them

1257 was present. The final prime motifs were established as the anchors. The flanking regions around 
1258 such anchored prime motifs were used to derive the contextual information, together which worked 1259 as freature vector elements for discrimination using XGBoost and Deep-Learning.

1261 Figure 2: The co-occurring motifs positional preference. The plots are showing the position specific 1262 existence of the co-occurring motifs with respect to the prime motif (coordinated at "0"). F-score 1263 values of other contextual features like position specific pentamers and heptamers distribution 1264 reflect this to some extent. Most of these RBPs exhibited some secondary motif which co-occured 1265 with the prime motif in a position specific manner.

1267 Figure 3: Comparison between experimentally reported motifs and motif identified in the present 1268 study. Most of the previously reported motifs for the RBPs were detected by the approach 1269 presented in the current study. However, it also observed that several of previously reported motifs 1270 are not the prime motifs but comparatively cover lesser CLIP-seq data than the prime motifs 1271 identified in the present study. The last three columns show the matching motifs similar to the 1272 previously reported motifs, their status in CLIP-seq data coverage, and the corresponding motif 1273 rank.

1275 Figure 4: F-score distribution of dinucleotide densities at different positional windows for the target 1276 regions and their flanks for Cluster\#1 members. Context specific dinucleotide density distribution 1277 emerged among the most important features for all RBPs taken in this study. Their densities worked 1278 as important features at variable windows and distances for different RBPs. Here, Cluster\# 1 1279 members data is shown. They shared high similarity among themselves for their prime binding 1280 motifs, yet their contextual information and density profiles differed a lot. Enriched contextual 1281 "CG” distribution of these regions was found consistently distinguished property for the regions 
1282 binding the RBPs.

1284 Figure 5: Assessment for three main properties in discriminating between the negative and positive 1285 instance,(A) Violin plot distribution of accuracy when dinucleotide, pentamer and heptamer were used alone for set A and set B RBP. (B) Impact of combination of the dinculetodie, pentamers, and heptamers properties based features. These features appeared highly additive, complementary to 1288 each other as the performance in accurately identifying the binding regions increases substantially 1289 as these are combined.

1291 Figure 6: Performance metrics for RBPSpot. (A) First plot showing the accuracy, AUC, sensitivity, specificity, F1 score for the DNN model for set A RBPs. The second plot is showing the same metrics for the gradient boosting method. The third plot is showing the corresponding instances in 1294 the test, train and in total data for set A RBPs, (B) The first plot is showing the accuracy, AUC, 1295 sensitivity,specificity, F1 score for the deep learning models for Set B RBPs, where the second plot 1296 is showing the same metrics values for the gradient boosting method with Set B RBPs. The third plot is showing the number of instances in the test, train and in total data for Set B RBPs. RBPSpot scored highly on all the performance metrics where the most remarkable thing about it was its consistent performance across a large number of RBPs and dataset.

Figure 7:AUC/ROC plots for Set A RBPs. The AUC/ROC plots for the deep-learning models for some of the RBPs clearly showcase the robustness and highly reliable performance of the implemented DNN models.

1305 Figure 8: Comparative bench-marking results of RBPSpot when compared to beRBP, DeepBind, 
1306 RBPmap, iDeepE, and DeepCLIP for three different datasets. (A) Bechmarking result on RBPSpot

1307 dataset, (B) Graphprot dataset, and (C) beRBP dataset. Each these datasets performances was

1308 evaluated for various performance metrics where the heatmaps are for accuracy, F-1 score, and

1309 MCC values for each dataset for some of the evaluated RBPs. The rightmost plots are radar charts

1310 view of the average Accuracy, F1 score, and MCC attained by each software for the corresponding

1311 dataset. The last plot is the box plot which provides the average distribution of these metrics scores.

1312 From the plots it is clearly visible that for all these datasets and for almost all of the RBPs, RBPspot

1313 consistently outperformed the compared tools for all the metrics. More all the radar plots it scored

1314 the highest and nearest to the isosceles triangle suggesting consistent and better average

1315 performance also. The box plot suggests that RBPSpot not only performed best in overall but also

1316 the dispersion of its various metric scores were much lesser than other compared tools. Some of

1317 these tools exhibited enormous variation in the distribution of their metric score suggesting unstable

1318 performance by them.

1320 Figure 9: Performance benchmarking with different combinations of train and test datasets. In this 1321 part of performance benchmarking the impact of datasets was also evaluated. Since this part 1322 required rebuilding of RBP-RNA interaciton models from the scratch and from the provided user 1323 defined data, only two other tools other than RBPSpot qualified this criteria (DeepCLIP and 1324 iDEEPE). These tools provide the capability to build new models from user given datasets. These 1325 tools were originally developed on Graphport dataset. Therefore, in this part of benchmarking 1326 RBPSpot and Graphprot datasets were considered and 4 different train-test datasets combinations 1327 were studied. Distributions for various performance metrics for the compared tools and the 1328 corresponding datasets have been given as Kernel densitiy plots: (A) RBPSpot train and test, (B) 1329 RBPSpot train and graphprot test, (C) Graphprot train and test, and (D) Graphprot train and 1330 RBPSpot test. For every such combinations, the average performance metics scores are given in the 
1331 form of heatmap (E). The plots clearly underline that RBPSpot consistently outperforms the two

1332 tools for all the metrics on all these different combinations of train and test datasets, where again the 1333 consistent and precise performance of RBPSpot was an important observation, Consistently high 1334 MCC scoring by RBPSpot underlined it as a robust and balanced algorithm where dispersion in 1335 performance metrics was least. Also, the performance of all the compared tools increased when 1336 RBPSpot dataset was used in training, clearly suggesting the importance of having a right dataset. 1337 RBPSpot dataset presented here emerged as a better dataset for such studies.

1339 Figure 10: Comparative time dependent root mean square deviations (RMSD) plots for 12 different 1340 RBP-RNA complexes of with and without the prime motif. The trajectory was measured at $300 \mathrm{~K}$ 1341 for the 20-ns. Trajectory arcs for RBP-complex of three randomly selected RNA sequences with 1342 motifs are shown in blue, green and violet spike arcs whereas trajectory spike-arcs for RBP1343 complex without motif were shown in orange, red and brown color. The complexes with the prime 1344 motifs were found much stable than their counterparts without the prime motif.

1346 Figure 11: Application of RBPSpot reports the binding sites for seven different RBP on nSARS1347 CoV2 genome. A total for 22 such binding sites were discovered across the nSARS-CoV2 genome, 1348 all which existed across the negative strand of the virus genome. 
bioRxiy preprint doi: https://doi.org/10.1101/2021.06.07.447370; this version posted June 7, 2021. The copyright holder for this preprinto of

which was not certified by peer review) is the author/funder, who has granted bioRxiv a license to display the preprint in perpetuity. It is

HNRNPK

$\begin{array}{rcc}\text { made available under aCC-BY } 4.0 \text { International license. } \\ \text { SERPSnt } & \text { SELEX/Y3H } & \text { Alignment }\end{array}$

RNACompete RBPSpot motif
/RIP-ChiP

support
Prime motif with prime motif

RBPSpot motif occurrence

matching to the experimentally reported motif if $\mathrm{R}$

of the matching motif from RBPSpot in CLIP-Seq instances

Rank of the matching RBPSpot motif

instances

$47.66 \%$

$11^{\text {th }}$

CCC

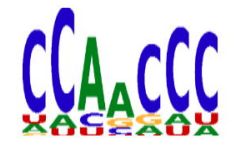

ACU

ACUAACA

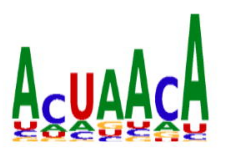

$62.74 \%$

$5^{\text {th }}$

ACU

Dseme

AUAAA

. | | | |

UUAAA

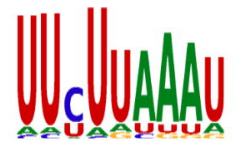

$70.48 \%$

$1^{\text {st }}$

UUUUG

| I. I

UUCUG

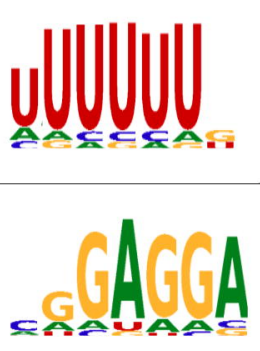

$59.68 \%$

$9^{\text {th }}$

TIA1

undous

Dou

LIN28A

CGAGG AUUCD

HNRNPC

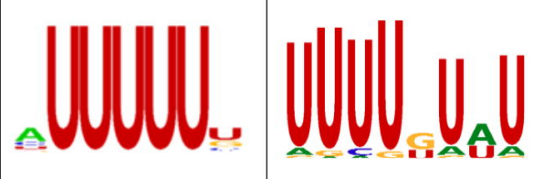

SRSF1

GGAGGA

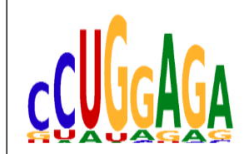

AGGACA

KHDRBS1

aldAAA

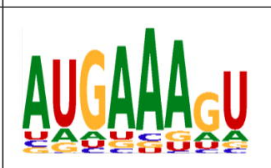

HNRNPA1

HNRNPL

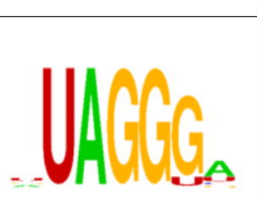

Ach CACA UUCACAd

KHDRBS2
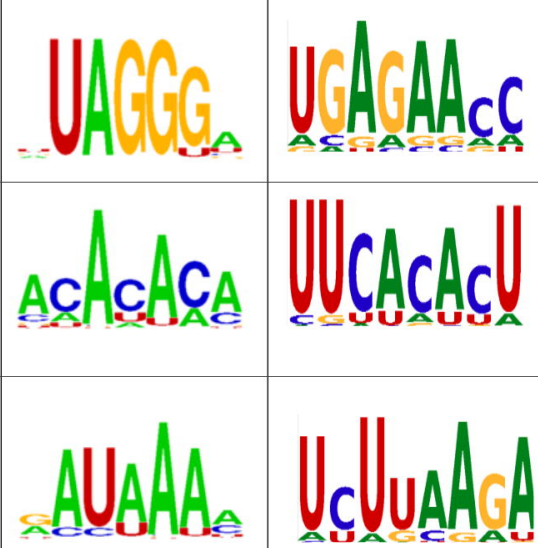

ACACACA

UUUUU

AGGACA

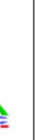

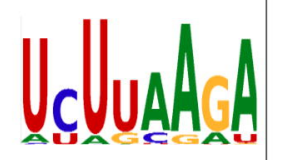

AAUAAAA

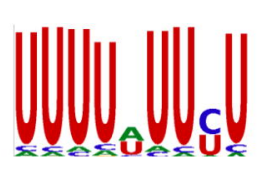

UUUUUU

TIAL1

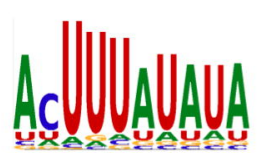

(n)


$\square$ DeepClip

(A)

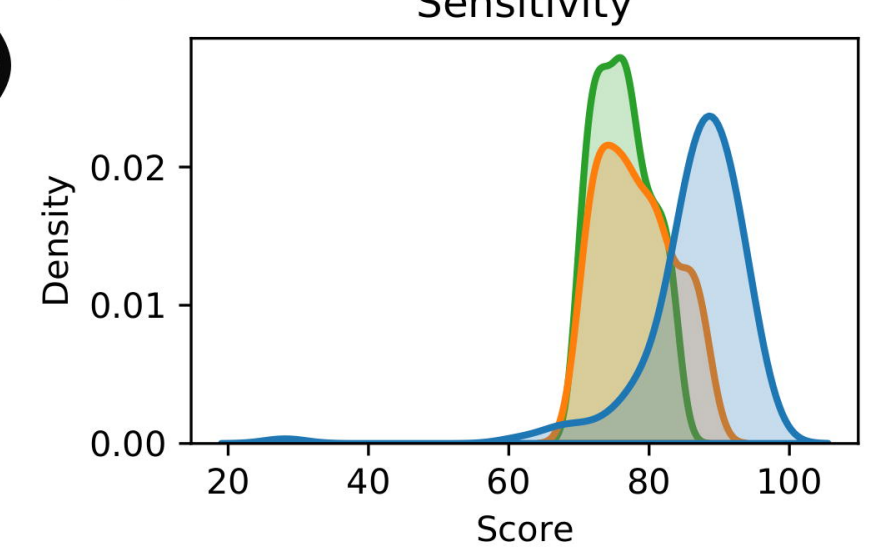

Sensitivity

(B)
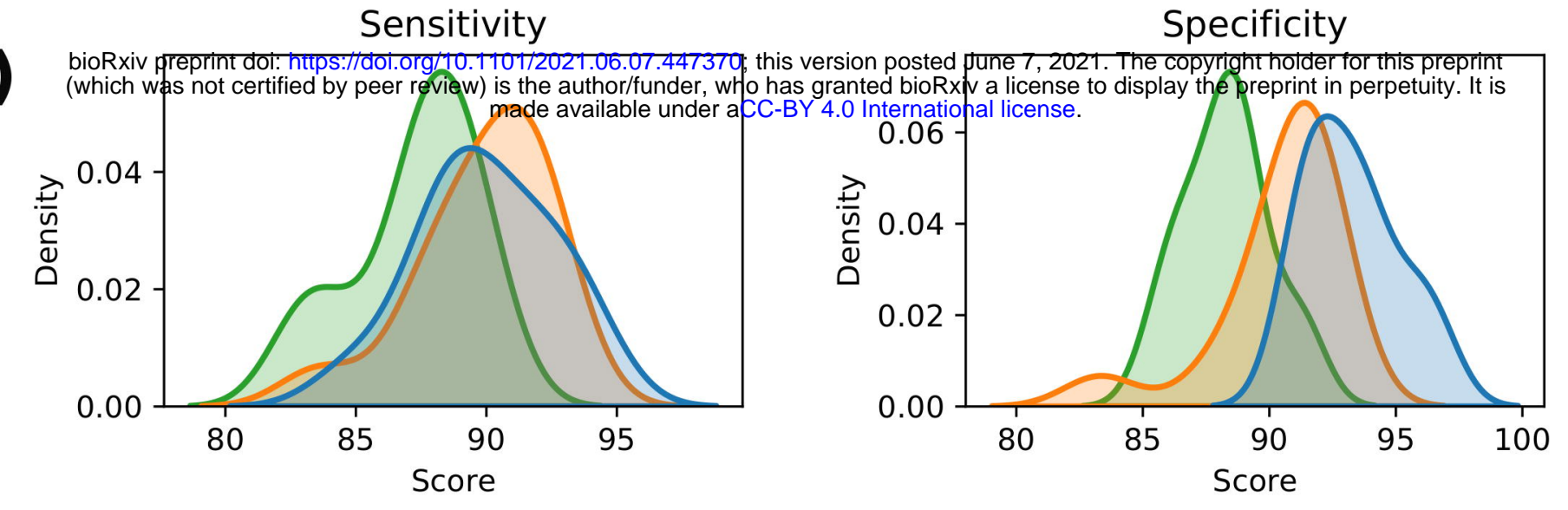

(C)
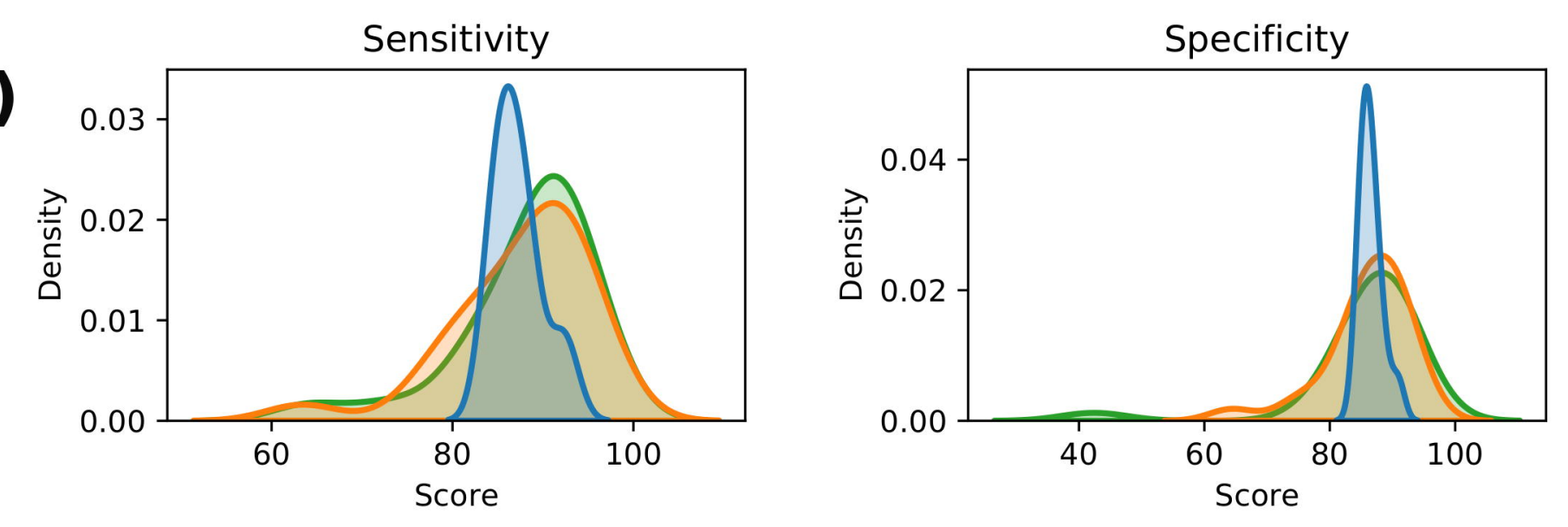

(D)

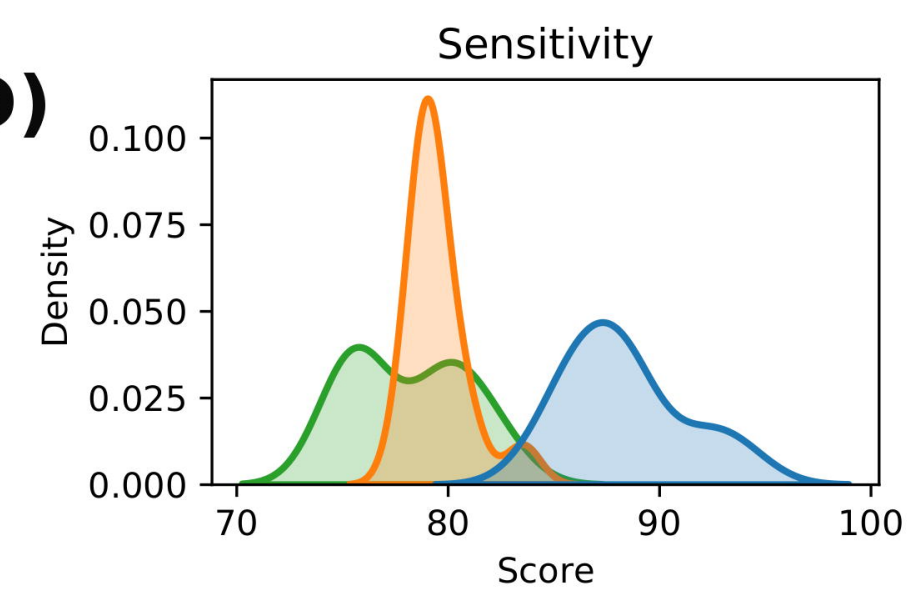

(E)

RBPSpot Train and Test

R日

$\frac{10}{20}$

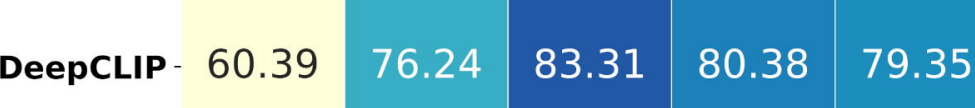

\begin{tabular}{ll|l|l|l|} 
IDeepE- 67.02 & 78.24 & 87.89 & 83.06 & 82.40
\end{tabular}

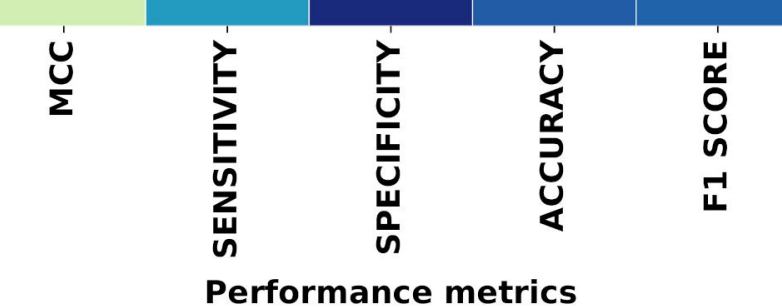

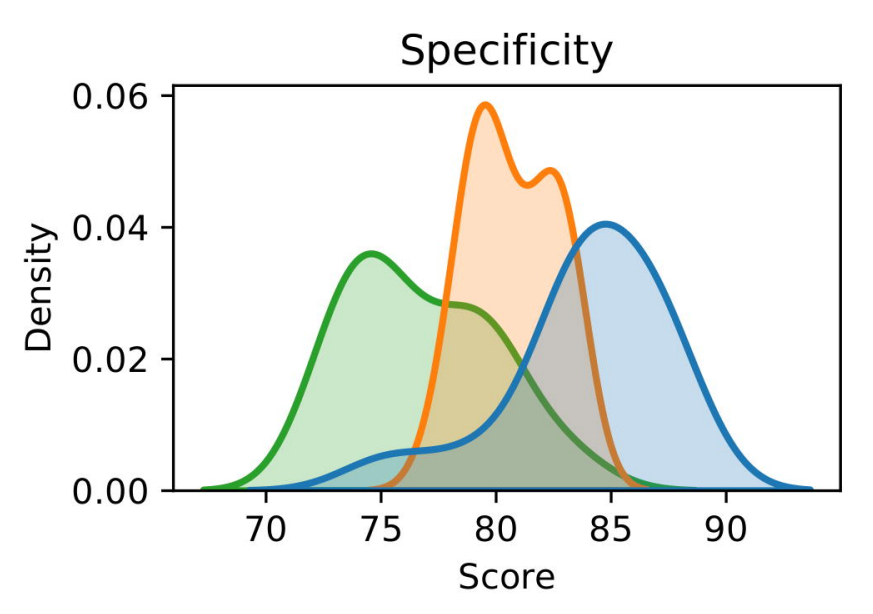

RBPSpot Train and Graph Prot Test \begin{tabular}{ll|l|l|l|l|} 
RBPSpot- 83.34 & 89.99 & 93.27 & 91.60 & 91.48
\end{tabular}

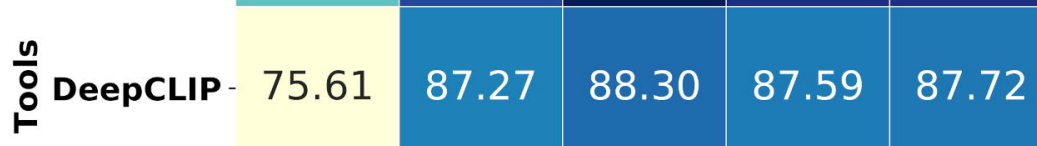
\begin{tabular}{ll|l|l|l} 
IDeepE- 80.39 & 89.89 & 90.46 & 90.18 & 90.15
\end{tabular}

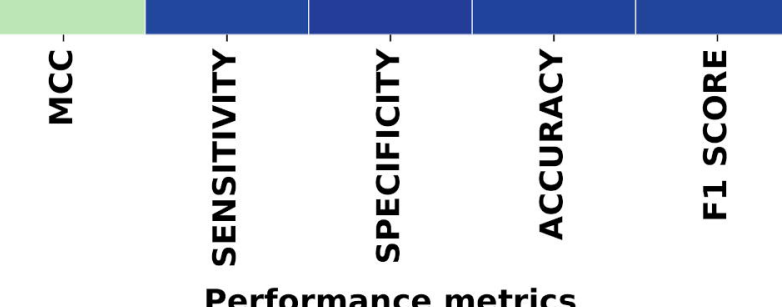
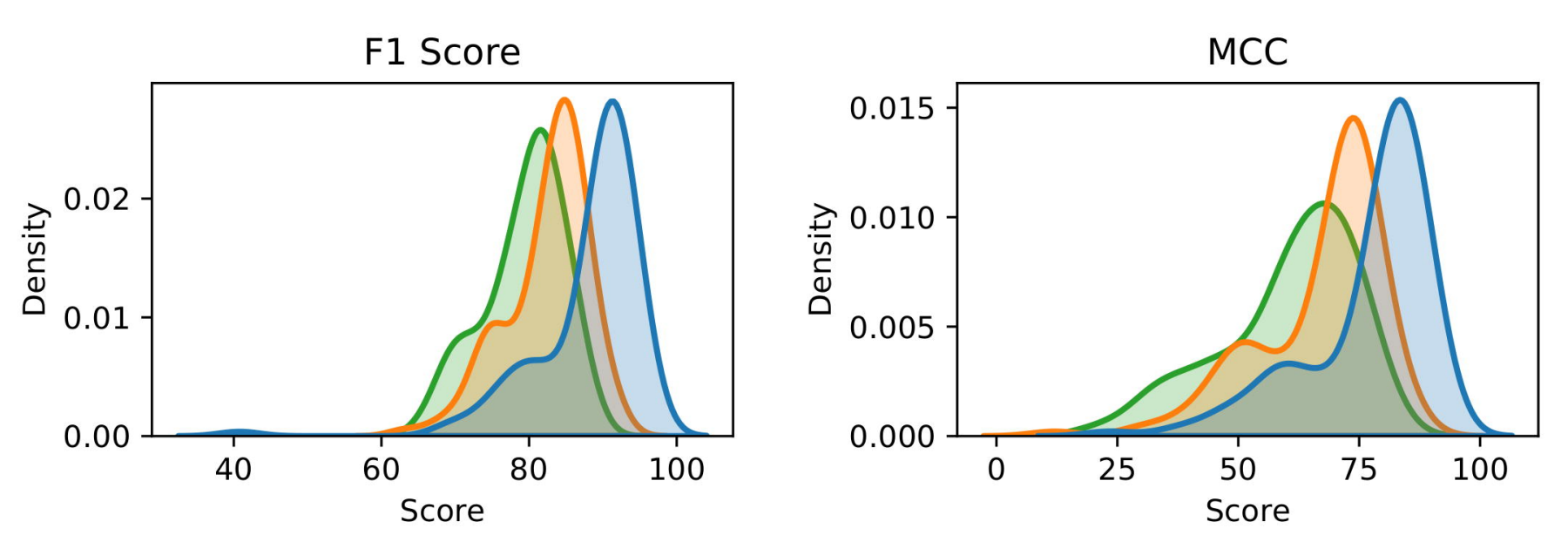

RBPSpot Train and Graph Prot Test
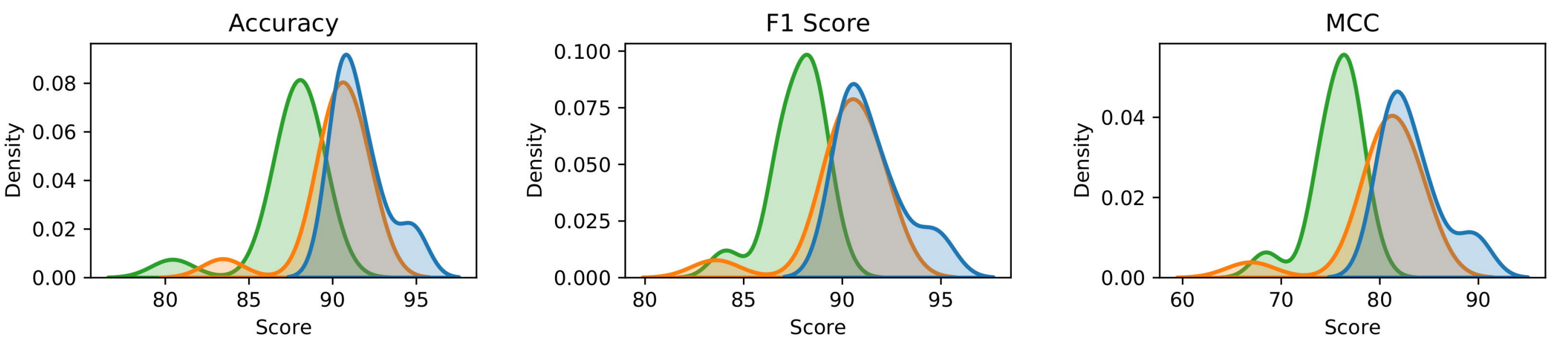

Graph Prot Train and Test
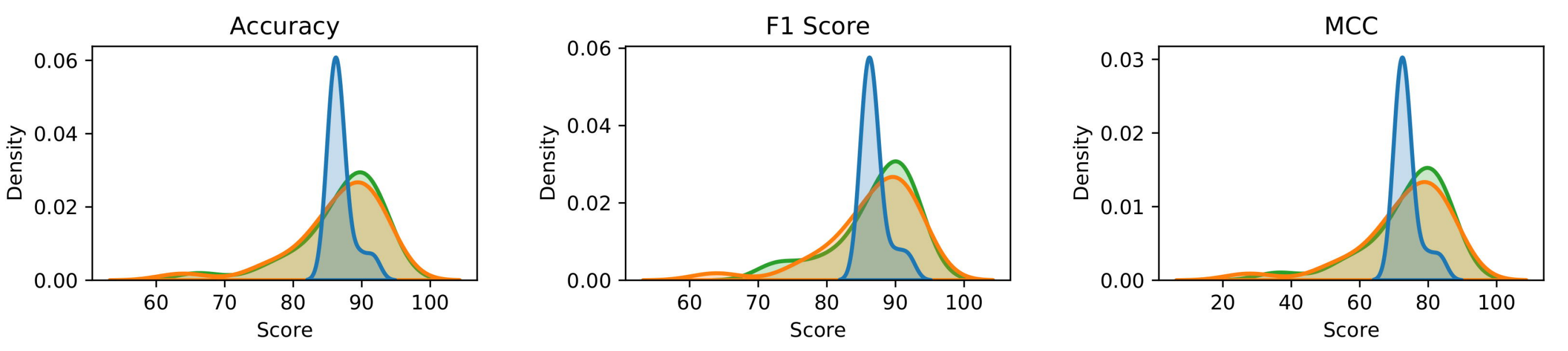

Graph Prot Train and RBPSpot Test
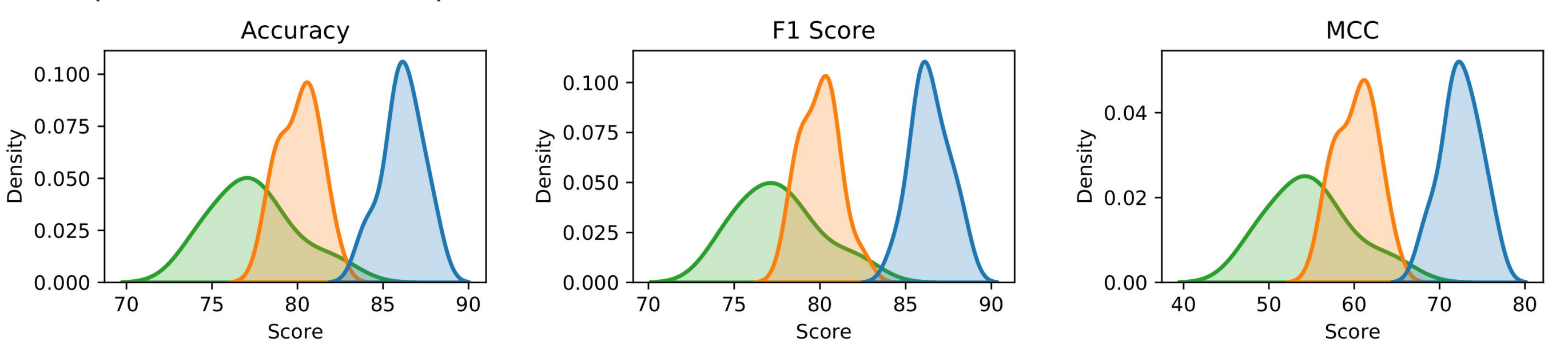

Graph Prot Train and RBPSpot Test
Graph Prot Train and Test

\begin{tabular}{l|l|l|l|l|} 
RBPSpot- 73.78 & 87.22 & 86.53 & 86.88 & 86.91 \\
\hline
\end{tabular}

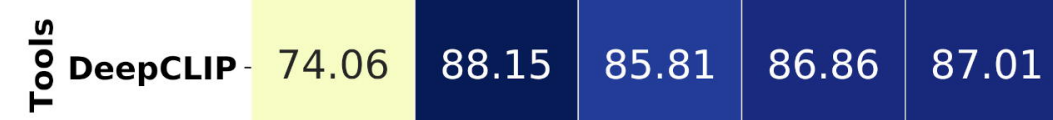

\begin{tabular}{ll|l|l|l|} 
IDeepE- 73.01 & 87.56 & 85.48 & 86.48 & 86.55
\end{tabular}

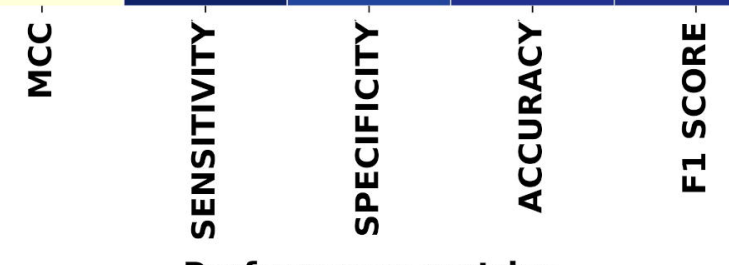

\begin{tabular}{ll|l|l|l|l|} 
RBPSpot & 72.47 & 88.34 & 83.95 & 86.14 & 86.44 \\
\hline
\end{tabular}

\begin{tabular}{l|l|l|l|l}
$\frac{n}{0}$ ODeepCLIP- 54.93 & 78.15 & 76.76 & 77.44 & 77.60 \\
\hline
\end{tabular}

\begin{tabular}{l|l|l|l|l} 
iDeepE- 60.24 & 79.53 & 80.72 & 80.11 & 79.99
\end{tabular}

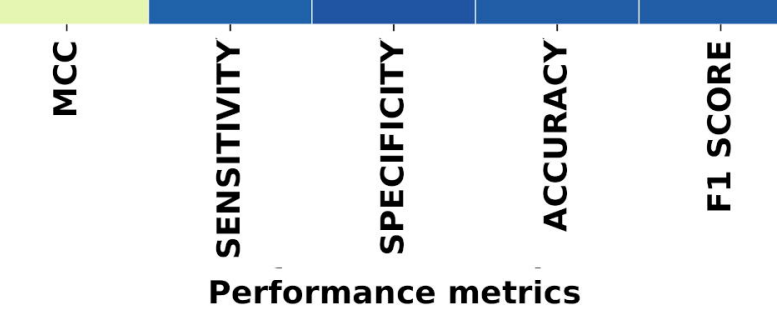




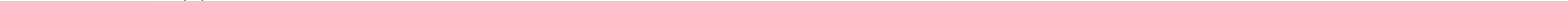




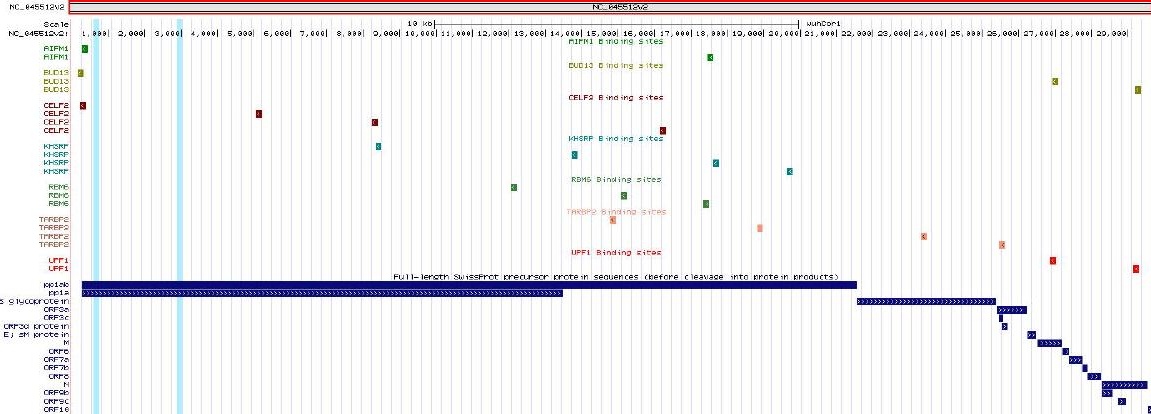

